TRANSACTIONS OF THE

AMERICAN MATHEMATICAL SOCIETY

Volume 355, Number 2, Pages 433-463

S 0002-9947(02)02986-0

Article electronically published on October 8, 2002

\title{
GROUP ACTIONS ON GRAPHS RELATED TO KRISHNAN-SUNDER SUBFACTORS
}

\author{
BINA BHATTACHARYYA
}

\begin{abstract}
We describe the principal graphs of the subfactors studied by Krishnan and Sunder in terms of group actions on Cayley-type graphs. This leads to the construction of a tower of tree algebras, for every positive integer $k$, which are symmetries of the Krishnan-Sunder subfactors of index $k^{2}$. Using our theory, we prove that the principal graph of the irreducible infinite depth subfactor of index 9 constructed by Krishnan and Sunder is not a tree, contrary to their expectations. We also show that the principal graphs of the Krishnan-Sunder subfactors of index 4 are the affine A and D Coxeter graphs.
\end{abstract}

\section{BACKGROUND AND InTRODUCTION}

Given a symmetric commuting square of finite dimensional $C^{*}$-algebras

$$
\begin{array}{llll} 
& B & \subset & D \\
\mathcal{C}: & \cup & & \cup \\
& A & \subset & C
\end{array}
$$

there is a well-known way to construct a subfactor $R_{\mathcal{C}} \subset R$ of the hyperfinite $\mathrm{II}_{1}$ factor from $\mathcal{C}$ (for commuting squares see [14, [4], [9]; for the construction see [19], 9]). This construction is quite general. A consequence of Popa's work is that any finite depth subfactor of the hyperfinite $\mathrm{II}_{1}$ subfactor can be constructed in this way $([15],[16],[13])$. However, the construction still keeps secrets. Ocneanu compactness (12], 9]) provides a method of computing the standard invariant of $R_{\mathcal{C}} \subset R$ and, in particular, the principal graph ([8], 4] ). (The principal graph $\Gamma$ is a possibly infinite graph with a distinguished root vertex $*$ and an eigenvector $\tau$ with eigenvalue the Jones index $\left[R: R_{\mathcal{C}}\right]$. It is a combinatoric encoding of the tower of higher relative commutants $\left(R_{\mathcal{C}}^{\prime} \cap R_{n}\right)_{n}$ where $R_{\mathcal{C}} \subset R_{0}=R \subset R_{1} \subset R_{2} \ldots$ is the Jones tower of $R_{\mathcal{C}} \subset R$.) Although in theory we can compute the $n$th higher relative commutant by solving a finite dimensional linear algebra problem, simply writing down the necessary equations takes time exponential in $n$, and the computation is in general intractable.

In [10], U. Krishnan and V. Sunder consider, in their words, "a seemingly simple class" of commuting squares. Let $k$ and $p$ be positive integers and $U$ a unitary matrix in $M_{p}(\mathbb{C}) \otimes M_{k}(\mathbb{C})$. A vertex model commuting square $([9])$ is a commuting

Received by the editors March 8, 1999 and, in revised form, December 17, 2001.

2000 Mathematics Subject Classification. Primary 46L37. 
square $\mathcal{C}$ of the form

$$
\begin{array}{ccc}
U\left(1 \otimes M_{k}(\mathbb{C})\right) U^{*} & \subset & M_{p}(\mathbb{C}) \otimes M_{k}(\mathbb{C}) \\
\cup & & \cup \\
\mathbb{C} & \subset & M_{p}(\mathbb{C}) \otimes 1
\end{array}
$$

Following Krishnan and Sunder's notation, we denote $R_{\mathcal{C}} \subset R$ by $R_{U} \subset R$ instead. The Jones index of $R_{U} \subset R$ is $k^{2}$. A Krishnan-Sunder subfactor is a subfactor $R_{U} \subset R$ for which $U$ is a permutation matrix. Krishnan and Sunder compute the principal graphs of all such subfactors of finite depth (i.e. $\Gamma$ is finite) in the case $k=p=2$ or $k=p=3$. In their analysis of Krishnan-Sunder subfactors they construct a discrete group $G$ and show that the vertices of $\Gamma$ correspond to finite dimensional representations of certain subgroups of $G$.

We add a new twist to Krishnan and Sunder's analysis of $R_{U} \subset R$ by constructing a graph $\mathcal{H}$ and a faithful action of $G$ on $\mathcal{H}$. The graph $\mathcal{H}$ has the property that each vertex of $\mathcal{H}$ is adjacent to exactly $k$ edges; also, $\mathcal{H}$ is infinite if and only if $R_{U} \subset R$ has infinite depth. We formulate $\Gamma$ in terms of $\mathcal{H}$ and the $G$-action as follows. Let $\left(P_{n}\right)_{n}$ be the tower of path algebras on $\mathcal{H}$ with the trace given by the constant weight vector on $\mathcal{H}$. Consider the subtower $\left(B_{n}\right)_{n}$ that commutes with the action of $G$ on $\mathcal{H}$. Then $\left(B_{n}\right)_{n}$ inherits Jones projections and a $k$-Markov trace from the path algebras. We show that there is a trace-preserving $*$-isomorphism of $\left(B_{n}\right)_{n}$ with the tower of higher relative commutants of $R_{U} \subset R$ that preserves the Jones projections. From this point it is straightforward to describe $\Gamma$ in terms of the representation theory of subgroups of $G$. As an example, we apply our theory to the case $k=2$ and show that the principal graphs of the Krishnan-Sunder subfactors of index 4 are $A_{2 n-1}^{(1)}, n \geq 1$, and $D_{n}^{(1)}, n \geq 4$. Our theory also yields a short proof of Theorem 37 in [10, which describes the principal graph of a particular infinite depth Krishnan-Sunder subfactor.

We remark that if $H$ and $\mathcal{G}$ are the group and graph of the index $p^{2}$ KrishnanSunder subfactor arising from (1.1), then $\mathcal{G}$ and $\mathcal{H}$ are bipartite generalizations of the Cayley graphs of $G$ and $H$, respectively (see Example 6.2 .2 of $[9$ for an example of this type of Cayley graph).

In 10], Krishnan and Sunder state that they believe the principal graphs of their two infinite depth subfactors are trees. We show that this is not the case, and in particular that the principal graph of the irreducible infinite depth subfactor is not a tree. We will show in a future note that the principal graph of the second infinite depth subfactor is not a tree either.

An outcome of our graphical formulation is the existence, for each $k$, of a tower of tree algebras $\left(T_{n}(k)\right)_{n}$ that are symmetries of all Krishnan-Sunder subfactors of index $k^{2}$. By this we mean they are contained in a canonical way in the tower of higher relative commutants of any Krishnan-Sunder subfactor of index $k^{2}$. We defined the tree algebras previously in [19] and showed in Corollary 6.2.5 and Corollary 6.2.6 of [19] that they are in fact a Popa system ( $\lambda$-lattice in [17]) and a sub-Popa system (or sub- $\lambda$-lattice) of the standard invariant of any Krishnan-Sunder subfactor of index $k^{2}$. Hence, the tree algebras $\left(T_{n}(k)\right)_{n}$ are themselves the higher relative commutants of a $\mathrm{II}_{1}$ subfactor of index $k^{2}$, which is irreducible, self-dual, and of infinite depth (Proposition 6.3.2 and Theorem 3.4.3 of [19]). However, the tree 
algebras are not the higher relative commutants of a Krishnan-Sunder subfactor (see Proposition 9.6).

This paper is organized into 11 sections. Sections 2 and 4 contain background and notation. General theory is developed in Section 3 and Sections 5 through 9 Sections 10 and 11 contain examples and calculations. The reader is encouraged to read the examples in parallel with the rest of the paper.

\section{ACKNOWLEDGEMENTS}

The content of Section 3 and the statement of Theorem 9.1 appear in my dissertation thesis, which I did at U. C. Berkeley. I would like to thank my advisor Vaughan Jones for directing me to Krishnan and Sunder's subfactors and for his many helpful comments. I would also like to thank David Evans, Viakalathur Sunder, and Fred Goodman for helpful suggestions during the research and writing of this paper. It is a pleasure to thank Roberto Longo at the University of Rome "Tor Vergata" and David Handelman and Thierry Giordano at the University of Ottawa for their hospitality during the research and writing of this paper.

\section{Notation And Jones Diagrams}

Let $V^{n}, n=0,1,2, \ldots$, be the complex vector space with orthonormal basis consisting of words of length $n$ on the alphabet $[k]\left(V^{0}=\mathbb{C} e\right.$, where $e$ is the empty word). We may identify $V^{n}$ with $\left(V^{1}\right)^{\otimes n}$ via the natural bijection of bases.

Then

$$
\mathbb{C} \cong \operatorname{End}\left(V^{0}\right) \subset \operatorname{End}\left(V^{2}\right) \subset \cdots \subset \operatorname{End}\left(V^{n}\right) \subset \ldots
$$

is a sequence of $*$-algebras with the inclusion map $F \mapsto F \otimes 1$. If $\operatorname{tr}_{n}$ is the unique trace on $\operatorname{End}\left(V^{n}\right)$ normalized so that $\operatorname{tr}_{n}(1)=1$, then $\operatorname{tr}_{n}$ respects the inclusion maps and extends to a trace tr on $\bigcup_{n} \operatorname{End}\left(V^{n}\right)$.

We briefly discuss the conventions of Jones diagrams, a convenient way to work with Ocneanu compactness [9]. Given $F \in \operatorname{End}\left(V^{n}\right)$, view $F$ as a matrix with respect to the basis $[k]^{n}$. Given a word $w \in[k]^{n}$, denote by $w^{t}$ the word $w$ spelled backwards. Denote the entry of $F$ in row $w_{b}=b_{1} b_{2} \ldots b_{n} \in[k]^{n}$ and column $w_{a}=a_{1} a_{2} \ldots a_{n} \in[k]^{n}$ by $F_{w}$, where $w=w_{b} w_{a}^{t}=b_{1} b_{2} \ldots b_{n} a_{n} \ldots a_{2} a_{1}$. We also represent this entry by a box surrounded by $2 n$ oriented strings labeled as below:

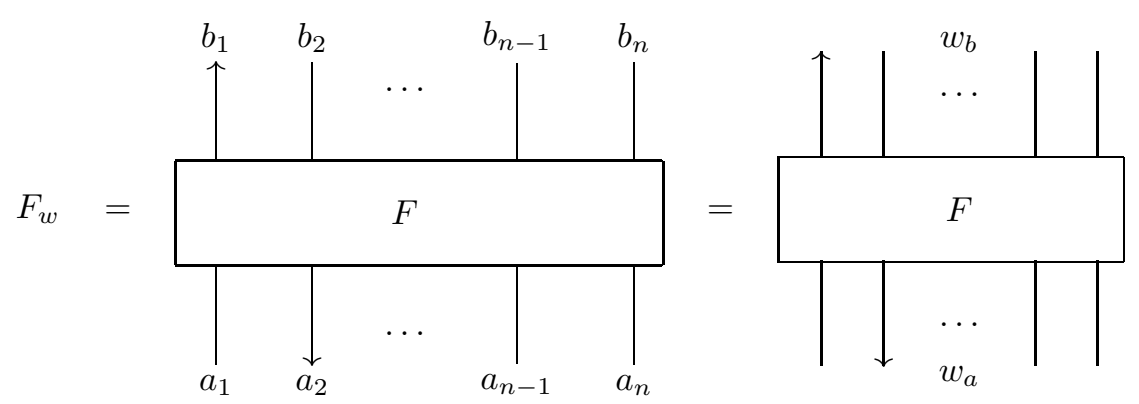

Let $U$ be a unitary matrix in $M_{p}(\mathbb{C}) \otimes M_{k}(\mathbb{C})$. Index the rows and columns of $U$ by the set $[p] \times[k]$, where $[p]$ is a set of $p$ elements and $[k]$ is a set of $k$ elements. Following Krishnan and Sunder's notation, we denote elements of $[p]$ 
by Greek letters and elements of $[k]$ by Roman letters. Denote the entry of $U$ in column $(\alpha, a)$ and row $(\beta, b)$ by $U_{\alpha a}^{\beta b}$. The square in (1.1) is a commuting square if and only if $U$ satisfies Ocneanu's biunitary condition ([13], [7]). In the vertex model case the biunitary condition on $U$ is that both $U$ and its "block transpose conjugate" $\widetilde{U} \in M_{p}(\mathbb{C}) \otimes M_{k}(\mathbb{C})$, defined by

$$
\widetilde{U}_{\beta a}^{\alpha b}=\overline{U_{\alpha a}^{\beta b}}
$$

are unitary. Such a $U$ is called a biunitary.

We represent the entries of $U$ and $\widetilde{U}$ by positive and negative labeled crossings:
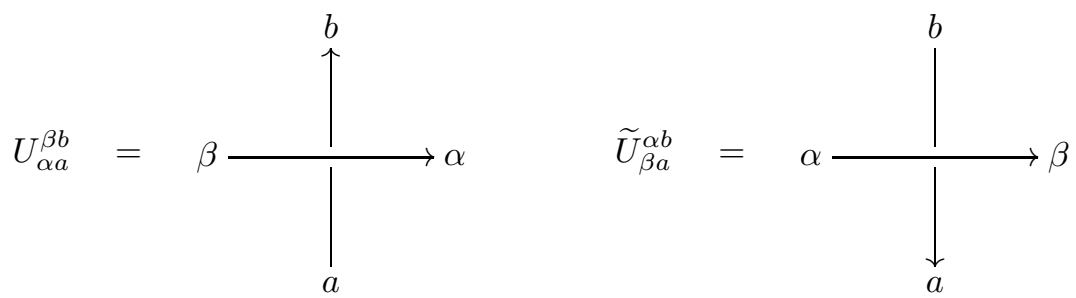

By definition of $\widetilde{U}$, the crossings above represent conjugate complex numbers.

Note that there are two types of strings, $[k]$-strings and $[p]$-strings, depending on the index set used. In a Jones diagram (such as the right-hand side of Figure 1 on the next page), the only allowed crossings are $[p]$-strings over $[k]$-strings. Strings are either bounded or unbounded; bits of string between crossings are considered bounded strings. A state on a diagram is a labeling of all strings from the appropriate index set, either $[k]$ or $[p]$. We evaluate a state by taking the product of all contributions from boxes and crossings. A diagram without any index labels is considered a function from its boundary conditions to $\mathbb{C}$. A boundary condition is a choice of indices for the unbounded strings, and it maps to the sum of the values of the states consistent with the boundary condition. For example, if $\gamma \in[p]$ and $b_{i}, a_{i} \in[k], 1 \leq i \leq 2 n$, then
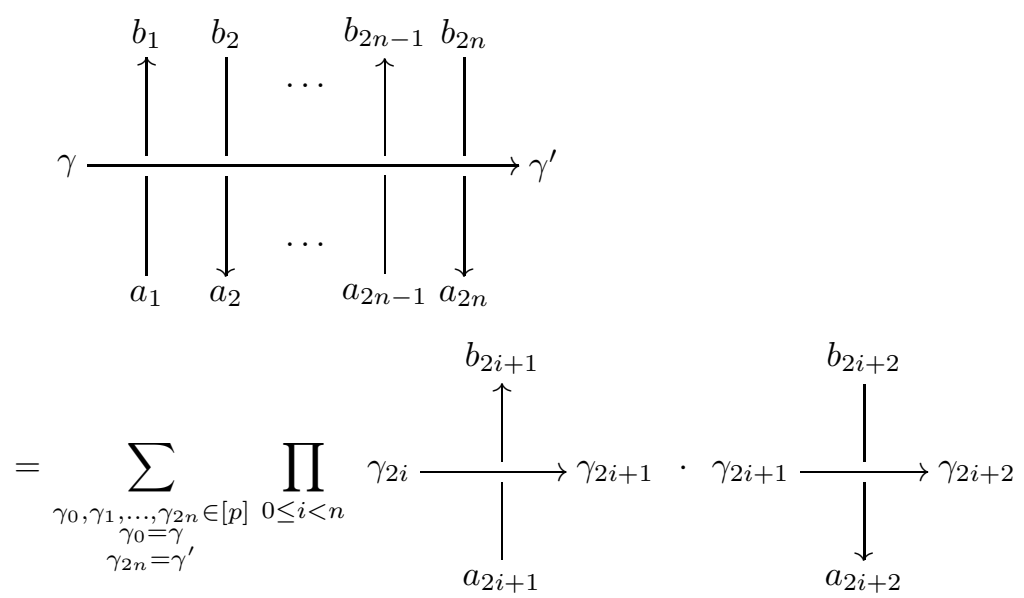
If $\alpha, \beta \in[p]$, the equation in Figure 1 translates as follows: for all $w \in[k]^{2 n}$,

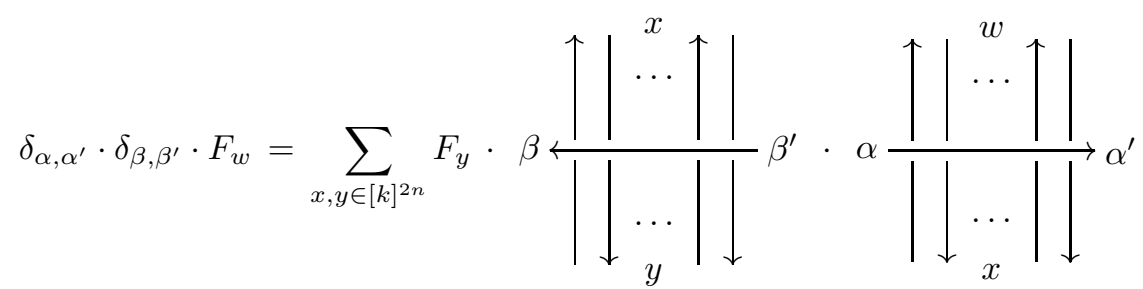

We say that $F \in \operatorname{End}\left(V^{n}\right)$ satisfies the relative commutant condition if for all $\alpha, \beta \in[p]$, Figure 1 holds. For $n=1,2, \ldots$, let

$$
\widetilde{B}_{n}=\left\{F \in \operatorname{End}\left(V^{n}\right): F \text { satisfies the relative commutant condition }\right\} \text {. }
$$

Set $\widetilde{B}_{0}=\operatorname{End}\left(V^{0}\right)=\mathbb{C} \cdot 1$. For each $n \geq 2$ define $\widetilde{E}_{n-1} \in \widetilde{B}_{n}$ by

$$
\left(\widetilde{E}_{n-1}\right)_{a_{1} a_{2} \ldots a_{2 n}}=\frac{1}{k} \delta_{a_{n-1}, a_{n}} \delta_{a_{n+1}, a_{n+2}} .
$$

Theorem 2.1 (Ocneanu, Jones). The sequence of $*$-algebras

$$
\mathbb{C}=\widetilde{B}_{0} \subset \widetilde{B}_{1} \subset \widetilde{B}_{2} \subset \cdots \subset \widetilde{B}_{n} \subset \cdots
$$

with the trace inherited from $\left(\operatorname{End}\left(V^{n}\right)\right)_{n}$ and the Jones projections $\widetilde{E}_{n}$ is isomorphic to the tower of higher relative commutants of $R_{U} \subset R$ ([9]).
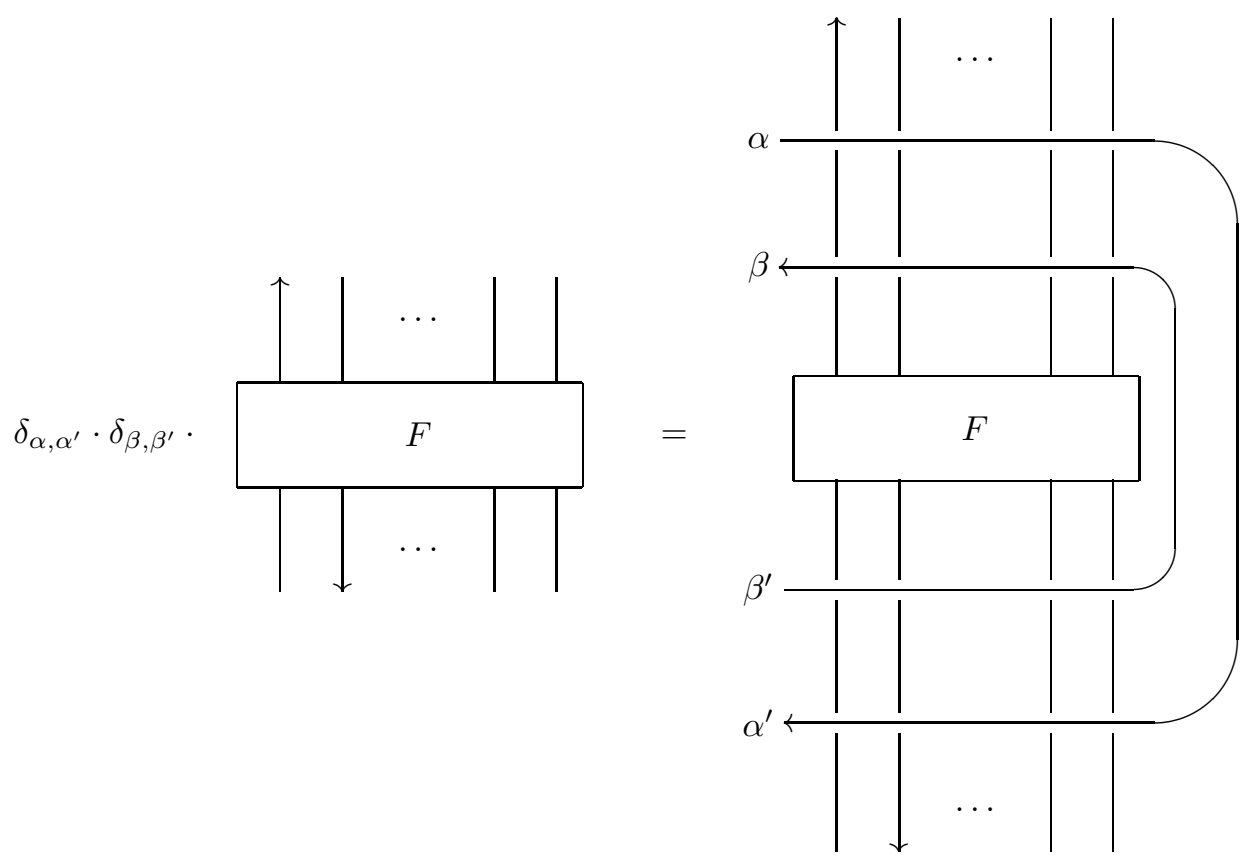

FiguRE 1. Relative commutant condition 


\section{The Group $G$}

Fix a permutation biunitary $U \in M_{p}(\mathbb{C}) \otimes M_{k}(\mathbb{C})$, that is, a biunitary whose entries are 0's and 1's. We give formulations of the $n$th higher relative commutant of $R_{U} \subset R$ in Proposition 3.5 and Corollary 3.6. These formulations are a twist on the one given by Krishnan and Sunder in [10].

Let $\mathcal{F}$ be the set of words of finite length on the alphabet $[k]$. The following is Proposition 6 in 10 .

Proposition 3.1 (Krishnan-Sunder). For each $w \in \mathcal{F}$, there exists a unique permutation action on $[p]$

$$
\begin{aligned}
w:[p] & \rightarrow[p] \\
\alpha & \mapsto w(\alpha)
\end{aligned}
$$

and for each $\alpha \in[p]$ a unique permutation action on $\mathcal{F}$

$$
\begin{aligned}
\alpha: \mathcal{F} & \rightarrow \mathcal{F} \\
w & \mapsto \alpha(w)
\end{aligned}
$$

such that

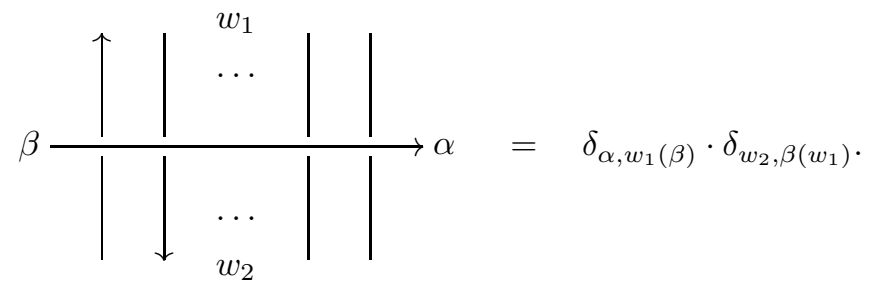

Remark 3.2. Noting that the crossings in (2.1) are either both equal to 1 or both equal to 0 , we have the following recursive formulas for the actions defined in Proposition 3.1. Let $\beta \in[p]$ and let $a_{1} a_{2} \ldots a_{r} a_{r+1}$ be a word of length $r+1>1$ in $\mathcal{F}$. Then

$$
a_{1} a_{2} \ldots a_{r+1}(\beta)= \begin{cases}a_{r+1}\left(a_{1} a_{2} \ldots a_{r}(\beta)\right), & \text { if } r \text { is even } \\ a_{r+1}^{-1}\left(a_{1} a_{2} \ldots a_{r}(\beta)\right), & \text { if } r \text { is odd }\end{cases}
$$

Also, define $\gamma \in[p]$ by

$$
\gamma= \begin{cases}a_{1} a_{2} \ldots a_{r}(\beta), & \text { if } r \text { is even } \\ a_{1} a_{2} \ldots a_{r} a_{r+1}(\beta), & \text { if } r \text { is odd }\end{cases}
$$

Then

$$
\beta\left(a_{1} a_{2} \ldots a_{r+1}\right)=\beta\left(a_{1} a_{2} \ldots a_{r}\right) \circ \gamma\left(a_{r+1}\right)
$$

where $\circ$ denotes concatenation of words.

Lemma 3.3. If $y, x \in \mathcal{F}$ have the same length and $\alpha, \beta \in[p]$, then

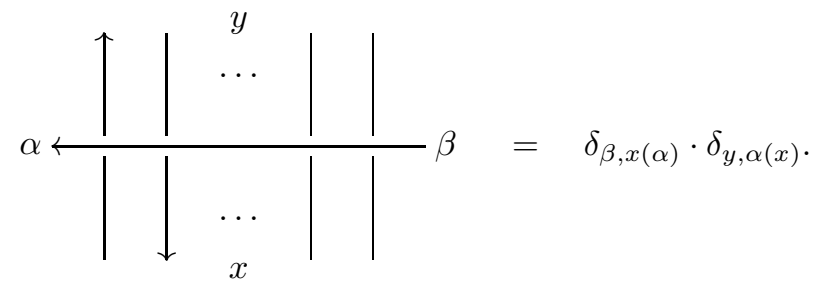


Proof. Set

$$
\begin{aligned}
& x^{\prime}=\alpha^{-1}(y), \\
& \beta^{\prime}=x^{\prime}(\alpha) .
\end{aligned}
$$

We have the following identity for Jones diagrams $[9$ :

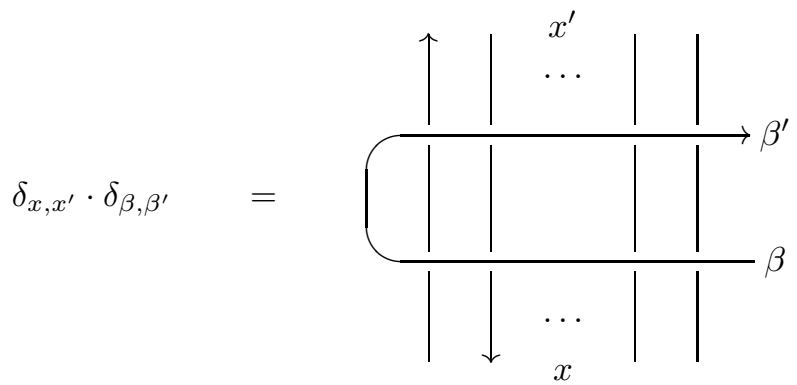

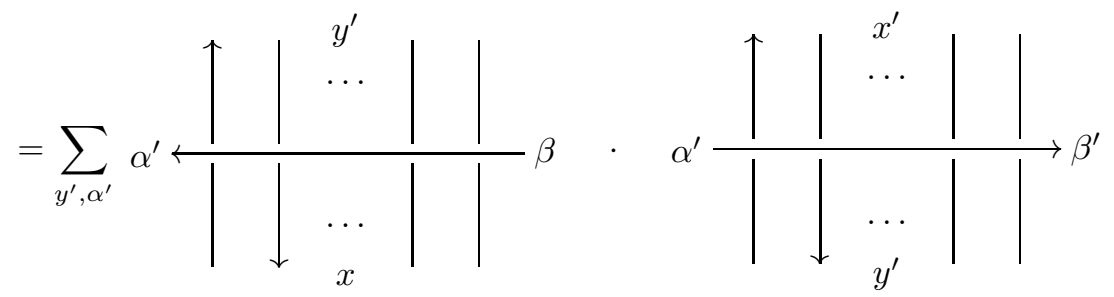

By Proposition 3.1 the second factor of (3.5) equals

$$
\delta_{\beta^{\prime}, x^{\prime}\left(\alpha^{\prime}\right)} \delta_{y^{\prime}, \alpha^{\prime}\left(x^{\prime}\right)}=\delta_{y, y^{\prime}} \delta_{\alpha, \alpha^{\prime}}
$$

Hence,

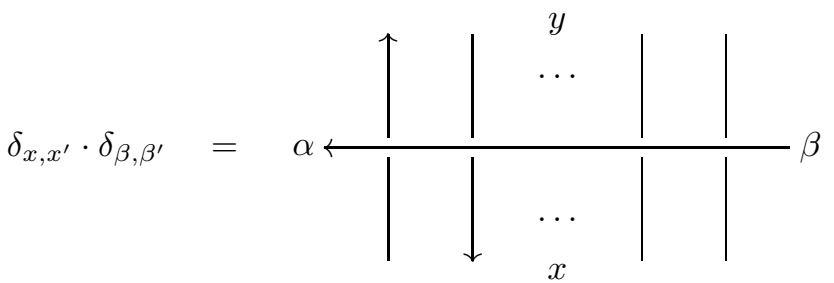

But

$$
\delta_{x, x^{\prime}} \cdot \delta_{\beta, \beta^{\prime}}=\delta_{y, \alpha(x)} \cdot \delta_{\beta, x(\alpha)}
$$

so we are done.

Action of $\mathcal{F}$ on $[p]^{2}$. For each $w \in \mathcal{F}$ define an action of $w$ on $[p]^{2}=[p] \times[p]$ by

$$
\begin{aligned}
w:[p]^{2} & \rightarrow[p]^{2} \\
(\alpha, \beta) & \mapsto w(\alpha, \beta)=\left(w(\alpha),\left(\beta^{-1} \alpha(w)\right)(\beta)\right) .
\end{aligned}
$$


Lemma 3.4. Let $w \in \mathcal{F}$ and $\alpha, \beta \in[p]$. Then

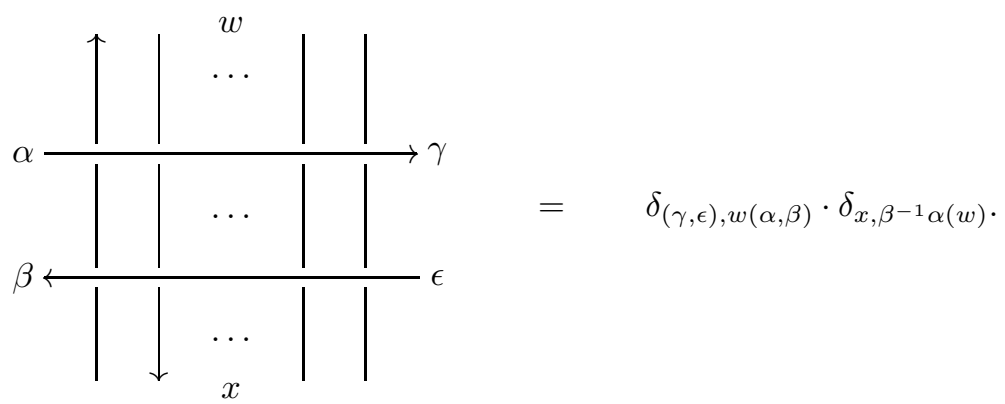

Proof. Let $n$ be the length of $w$. The left-hand side of (3.6) equals

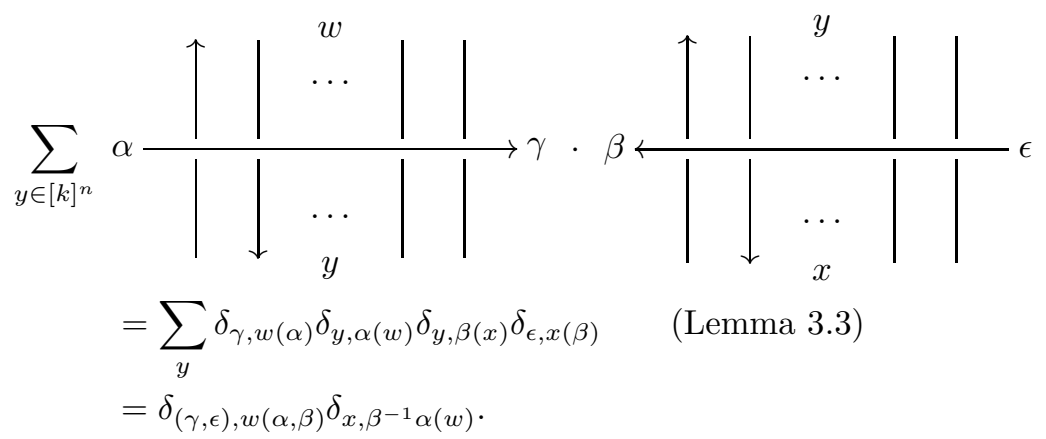

The following proposition and corollary reformulate Lemma 8 and part of Proposition 10 in [10.

Proposition 3.5. The following are necessary and sufficient conditions for $F \in$ $\operatorname{End}\left(V^{n}\right)$ to satisfy the relative commutant condition. For all $\alpha, \beta \in[p]$ and $w \in \mathcal{F}$ of length $2 n$,

(1) if $F_{w} \neq 0$, then $w$ acts identically on $[p]^{2}$;

(2) $F_{w}=F_{\beta^{-1} \alpha(w)}$.

Proof. Using the relative commutant condition in Figure 1 and unwrapping the unitary lines, we obtain the equivalent condition: for all $\alpha, \alpha^{\prime}, \beta, \beta^{\prime} \in[p]$,

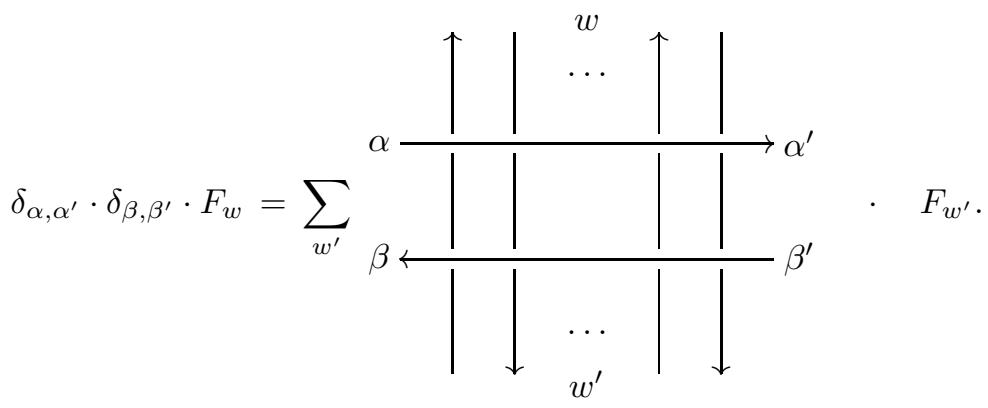

By Lemma 3.4 (3.7) is equivalent to

$$
\delta_{\alpha, \alpha^{\prime}} \cdot \delta_{\beta, \beta^{\prime}} \cdot F_{w}=\delta_{\left(\alpha^{\prime}, \beta^{\prime}\right), w(\alpha, \beta)} F_{\beta^{-1} \alpha(w)} .
$$


The conditions of the proposition clearly imply (3.8) for all $\alpha, \alpha^{\prime}, \beta, \beta^{\prime}, w$. We must prove necessity of the conditions, assuming (3.8) holds for all $\alpha, \beta, w$. Set $\alpha^{\prime}=\alpha$ and $\beta^{\prime}=\beta$. Then the first condition is obvious. The second condition is obvious if $w(\alpha, \beta)=(\alpha, \beta)$. If $w(\alpha, \beta) \neq(\alpha, \beta)$, then $F_{w}=0$, and by symmetry

$$
F_{\beta^{-1} \alpha(w)}=\delta_{(\beta, \alpha), \beta^{-1} \alpha(w)(\beta, \alpha)} F_{w} .
$$

Therefore, $F_{\beta^{-1} \alpha(w)}=0$ as well.

The Group of $R_{U} \subset R$. As in [10], define $G$ to be the subgroup of permutations of $\mathcal{F}$ generated by the elements $\beta^{-1} \alpha$, for $\alpha, \beta \in[p]$.

Corollary 3.6. The following are necessary and sufficient conditions for $F \in$ $\operatorname{End}\left(V^{n}\right)$ to satisfy the relative commutant condition. For all $w \in \mathcal{F}$ of length $2 n$,

(1) if $F_{w} \neq 0$, then for all $g \in G, g(w)$ acts identically on $[p]^{2}$;

(2) for all $g \in G, F_{w}=F_{g(w)}$.

Proof. It is easy to see that these conditions are equivalent to the conditions of the previous proposition.

Remark 3.7. By the definition of the action of $\mathcal{F}$ on $[p]^{2}, g(w)$ acts identically on $[p]^{2}$ for all $g \in G$ iff $g(w)$ acts identically on $[p]$ for all $g \in G$.

\section{Notation for Graphs}

We define a graph $\mathcal{H}=(V, E)$ to be a pair of sets $V$ and $E$, the vertex set and the edge set respectively, such that to every edge $e \in E$ there are associated two vertices in $V$, called its endpoints. Thus our graphs have unoriented edges and allow multiple edges. In fact, all the graphs we consider are bipartite, so in particular we will not consider graphs with loops. A homomorphism of graphs

$$
\phi: \mathcal{H}_{1}=\left(V_{1}, E_{1}\right) \rightarrow \mathcal{H}_{2}=\left(V_{2}, E_{2}\right)
$$

consists of maps of sets $\phi_{V}: V_{1} \rightarrow V_{2}$ and $\phi_{E}: E_{1} \rightarrow E_{2}$ such that if $\phi_{E}: e \mapsto f$, then $\phi_{V}$ maps the endpoints of $e$ onto the endpoints of $f$.

A path $\rho$ on $\mathcal{H}$ of length $n$ is a finite alternating sequence of vertices and edges, $v_{0} e_{1} v_{1} e_{2} \ldots e_{n} v_{n}$, where $v_{i} \in V, 0 \leq i \leq n$, and $e_{i} \in E, 1 \leq i \leq n$, such that for each $i$ the set of endpoints of $e_{i}$ is $\left\{v_{i-1}, v_{i}\right\}$. We say that $e_{i}$ is between $v_{i-1}$ and $v_{i}$. We say that $\rho$ starts at $v_{0}$ and ends at $v_{n}$. Denote by $\rho^{t}$ the path

$$
v_{n} e_{n} v_{n-1} e_{n-1} \ldots e_{1} v_{0}
$$

If $\mathcal{H}$ has a distinguished root vertex, then the path $\rho$ is a rooted path if it starts at the root. We will denote the root vertex of a graph by $*$. If $\rho_{1}$ and $\rho_{2}$ are two paths 
and $\rho_{2}$ starts at the vertex at which $\rho_{1}$ ends, we denote the concatenation of $\rho_{1}$ and $\rho_{2}$ by $\rho_{1} \circ \rho_{2}$. The distance between two vertices $v$ and $w$ is the minimum path length over all paths starting at $v$ and ending at $w$. Vertices $v$ and $w$ are neighbors if the distance between them is 1 .

In Section 9] we will want to treat graphs as topological spaces. A graph $\mathcal{H}=$ $(V, E)$ can be represented by a topological space $\mathcal{H}^{r}$ as defined in [6], p. 17. Each vertex is represented by a distinct point and each edge is represented by a distinct arc homeomorphic to the closed interval $[0,1]$. The boundary points of an arc represent the endpoints of the corresponding edge. The interiors of the arcs are mutually disjoint and do not meet the points representing vertices. If $\phi: \mathcal{H}_{1} \rightarrow \mathcal{H}_{2}$ is a graph morphism, then the induced topological map $\phi^{r}: \mathcal{H}_{1}^{r} \rightarrow \mathcal{H}_{2}^{r}$ is continuous.

Given a vertex $v$ of $\mathcal{H}$, let $E_{\mathcal{H}}(v)$ denote the set of edges which have $v$ as an endpoint. Following [3], we call a graph morphism $\phi: \mathcal{H}_{1} \rightarrow \mathcal{H}_{2}$ a local isomorphism if for all vertices $v$ in $\mathcal{H}_{1}$, the restriction of $\phi$ to $E_{\mathcal{H}_{1}}(v)$ is a bijection of $E_{\mathcal{H}_{1}}(v)$ with $E_{\mathcal{H}_{2}}(\phi(v))$.

Lemma 4.1. $\phi^{r}: \mathcal{H}_{1}^{r} \rightarrow \mathcal{H}_{2}^{r}$ is a covering map if $\phi: \mathcal{H}_{1} \rightarrow \mathcal{H}_{2}$ is a surjective local isomorphism.

Proof. See 6], p. 75. A similar result is proved in [3].

\section{The GRAPH $\mathcal{H}$}

An important ingredient of Krishnan and Sunder's analysis is an equivalence relation on words in $\mathcal{F}$. The main point of this section is to encode that relation into a graph $\mathcal{H}$. For this purpose it is more convenient to define a superficially different relation in Definition [5.3, but we prove in Proposition 5.6 that the two relations are essentially the same.

Lemma 5.1. Let $w_{1}, w_{2} \in \mathcal{F}$ be words of the same length and let $\alpha, \beta, \gamma, \delta \in[p]$. Then
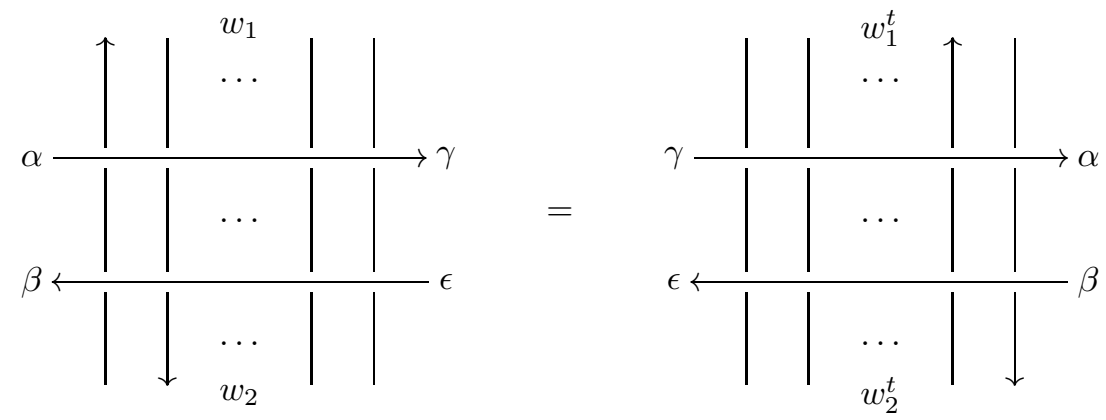
Proof. Rotating the right-hand side of (5.1) by 180 degrees, we obtain

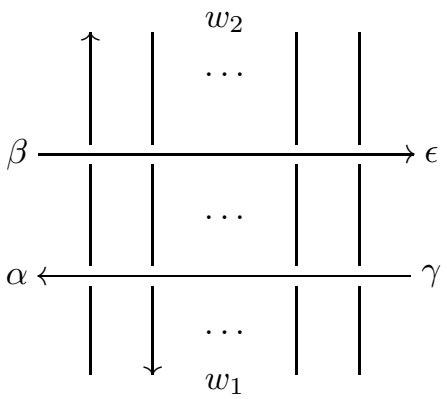

which equals (using Lemma 3.3 and Proposition 3.1 to deduce (5.3)
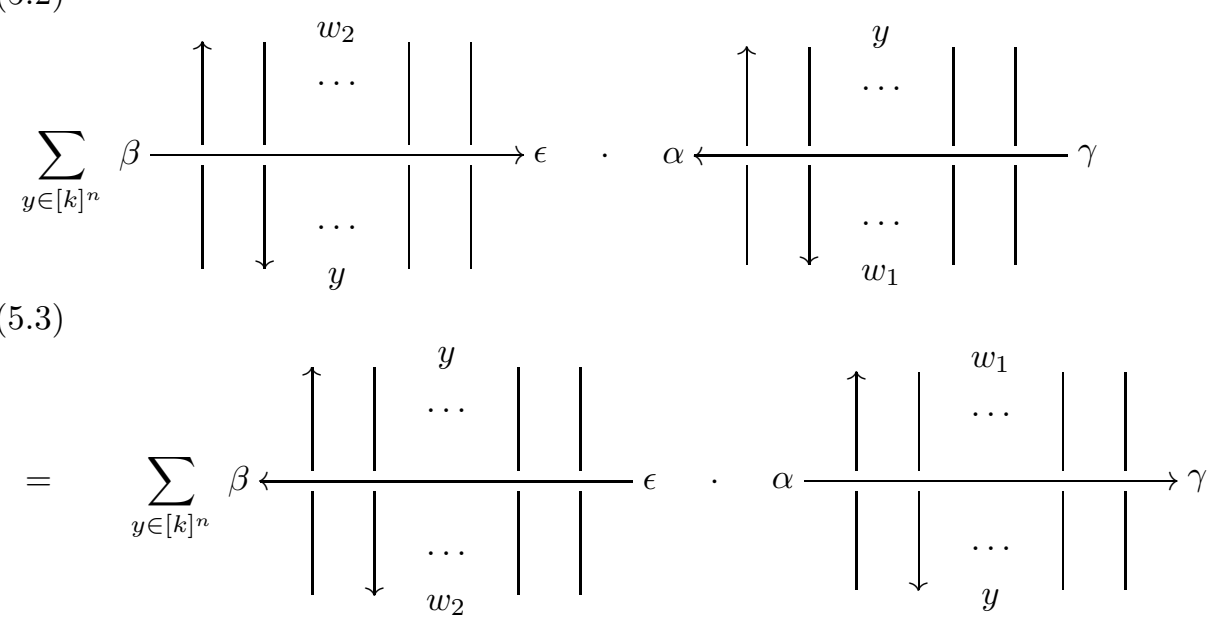

which equals the left-hand side of (5.1).

Lemma 5.2. Assume $w_{1}, w_{2} \in \mathcal{F}$ and $w_{1} w_{2}^{t}$ has even length.

(1) Let $\alpha, \beta \in[p]$ and set $g=\beta^{-1} \alpha \in G$. If $w_{1} w_{2}^{t}$ acts identically on $[p]^{2}$, then $g\left(w_{1} w_{2}^{t}\right)=g\left(w_{1}\right) g\left(w_{2}\right)^{t}$.

(2) $w_{1}$ and $w_{2}$ have the same action on $[p]^{2}$ if and only if $w_{1} w_{2}^{t}$ acts identically on $[p]^{2}$.

Proof. Fix any $\alpha, \beta \in[p]$. Let $(\gamma, \epsilon)=w_{1}(\alpha, \beta)$, and let $x_{1}$ and $x_{2}$ be words in $\mathcal{F}$ of the same length as $w_{1}$ and $w_{2}$, respectively. We have (using Lemma 3.4 to deduce 
(5.5) and Lemma 5.1 to deduce (5.61))

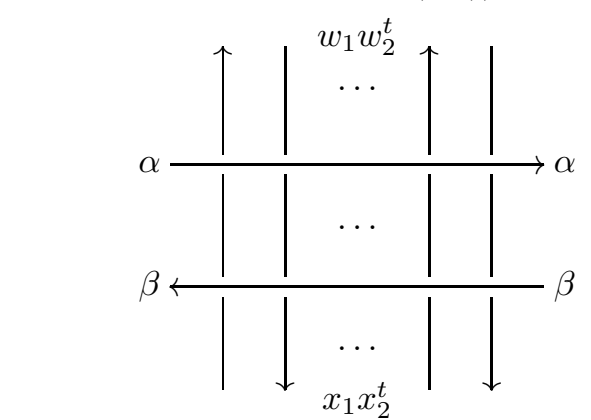

$$
(5.6)=
$$
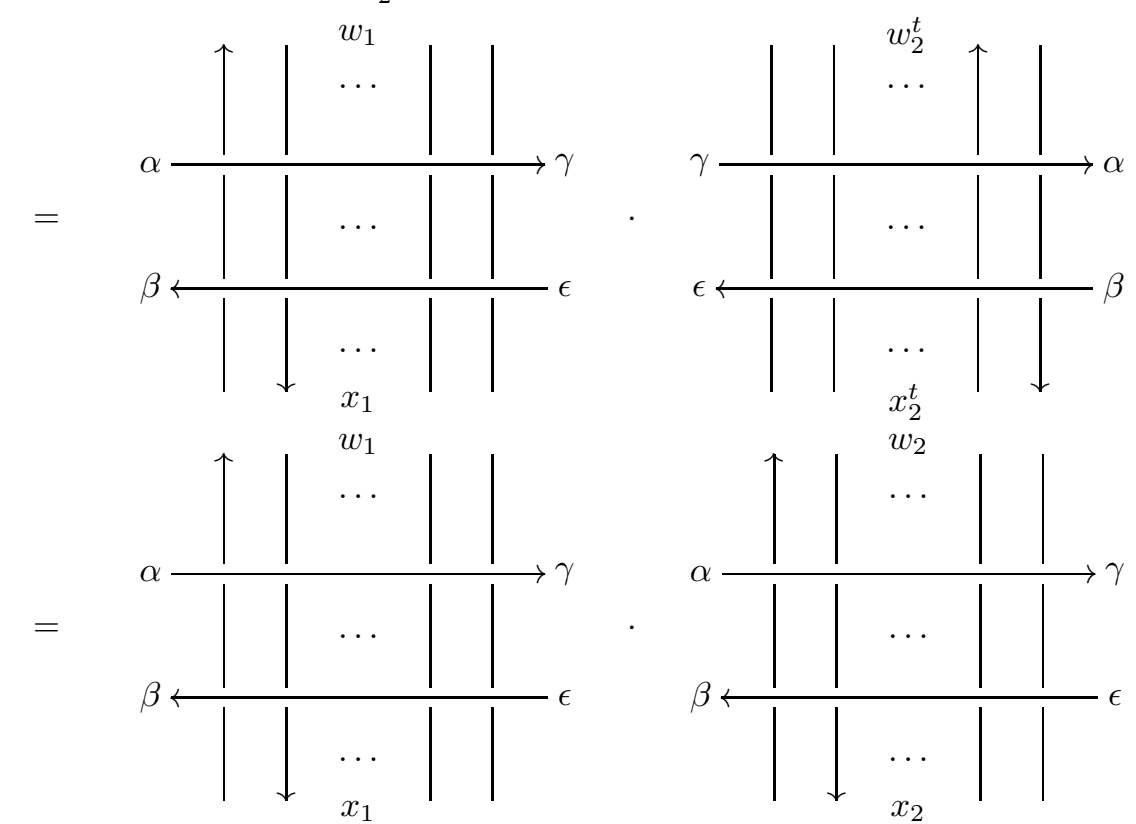

Assume that $w_{1}$ and $w_{2}$ have the same action on $[p]^{2}$, and set $x_{1}=\beta^{-1} \alpha\left(w_{1}\right)$ and $x_{2}=\beta^{-1} \alpha\left(w_{2}\right)$. By Lemma 3.4 (5.6) equals 1. Applying Lemma 3.4 to (5.4), we obtain that $w_{1} w_{2}^{t}$ acts identically on $(\alpha, \beta)$. Since $(\alpha, \beta)$ was arbitrary, this proves direction ( $\Longrightarrow$ ) of part 2 .

Now assume that $w_{1} w_{2}^{t}$ acts identically on $[p]^{2}$. Let $x_{1}$ and $x_{2}$ be such that $x_{1} x_{2}^{t}=\beta^{-1} \alpha\left(w_{1} w_{2}^{t}\right)$. By Lemma 3.4. (5.4) equals 1. Applying Lemma 3.4 to (5.61), we obtain $w_{2}(\alpha, \beta)=(\gamma, \epsilon), x_{1}=\beta^{-1} \alpha\left(w_{1}\right)$, and $x_{2}=\beta^{-1} \alpha\left(w_{2}\right)$. Then $w_{1}(\alpha, \beta)=w_{2}(\alpha, \beta)$ for arbitrary $(\alpha, \beta)$, which finishes the proof of part 2. And $g\left(w_{1} w_{2}^{t}\right)=g\left(w_{1}\right) g\left(w_{2}\right)^{t}$, which proves part 1 .

The following relation is essentially the same as the one defined by Krishnan and Sunder in [10], as we shall see in Proposition [5.6.

Definition 5.3. Define the relation $\sim$ on $\mathcal{F}$ by $w_{1} \sim w_{2}$ if $w_{1} w_{2}^{t}$ is of even length and if for all $g \in G, g\left(w_{1} w_{2}^{t}\right)$ acts identically on $[p]^{2}$. We may also write $w_{1} w_{2}^{t} \sim e$, where $e$ denotes the empty word.

Lemma 5.4. The set $\{w \in \mathcal{F}: w \sim e\}$ is stabilized by $G$.

Proof. Obvious. 
Lemma 5.5. Let $w_{1}, w_{2} \in \mathcal{F}$. If $w_{1} \sim w_{2}$, then for all $g \in G, g\left(w_{1} w_{2}^{t}\right)=$ $g\left(w_{1}\right) g\left(w_{2}\right)^{t}$.

Proof. Using Lemma 5.4 and Lemma 5.2, this is an exercise in induction on the number of generators needed to generate $g$.

Proposition 5.6. For all $w_{1}, w_{2} \in \mathcal{F}, w_{1} \sim w_{2}$ if and only if $w_{1} w_{2}^{t}$ has even length and for all $g \in G, g\left(w_{1}\right)$ and $g\left(w_{2}\right)$ have the same action on $[p]^{2}$.

Proof. Direction $(\Rightarrow)$ follows immediately from Lemma [5.5, Lemma 5.4, and part 2 of Lemma 5.2. To prove the other direction, assume that for all $g \in G, g\left(w_{1}\right)$ and $g\left(w_{2}\right)$ have the same action on $[p]^{2}$. Then for all $g, g\left(w_{1}\right) g\left(w_{2}\right)^{t}$ acts identically on $[p]^{2}$ by Lemma 5.2 And $g^{-1}\left(g\left(w_{1}\right) g\left(w_{2}\right)^{t}\right)=w_{1} w_{2}^{t}$ by the first part of Lemma 5.2 hence $g\left(w_{1} w_{2}^{t}\right)=g\left(w_{1}\right) g\left(w_{2}\right)^{t}$. Therefore $g\left(w_{1} w_{2}^{t}\right)$ acts identically on $[p]^{2}$ for all $g \in G$, so $w_{1} \sim w_{2}$.

Corollary 5.7. The relation $\sim$ is an equivalence relation.

Proof. Immediate from Proposition [5.6.

The Graph of $R_{U} \subset R$. We can now construct the rooted graph associated to the Krishnan-Sunder subfactor $R_{U} \subset R$. The graph $\mathcal{H}=\left(V_{U}, E_{U}\right)$ associated to $R_{U} \subset R$ has vertex set $V_{U}=\mathcal{F} / \sim$, the set of equivalence classes of $\mathcal{F}$. We denote by $[w]$ the equivalence class of $w \in \mathcal{F}$. The edge set of $\mathcal{H}$ is

$$
E_{U}=\left\{\left(\left\{\left[w_{u}\right],\left[w_{v}\right]\right\}, a\right): w_{u}, w_{v} \in \mathcal{F}, a \in[k], \text { and } w_{u} a w_{v}^{t} \sim e\right\}
$$

which we show to be well-defined in the following lemma. The endpoints of the edge $(\{u, v\}, a)$ in $E_{U}$ are $u$ and $v$. The root of $\mathcal{H}$ is the equivalence class of the empty word $e$. Note that $\mathcal{H}$ is bipartite: the even vertices are those classes whose representatives have even length. Define the weight vector $\tau$ on $\mathcal{H}$ by $\tau_{u}=1$ for every vertex $u$ of $\mathcal{H}$. We shall see in Remark 5.9 that $\tau_{U}$ is an eigenvector of $\mathcal{H}$ with eigenvalue $k$.

Lemma 5.8. The condition $w_{u} a w_{v}^{t} \sim e$ is independent of the choice of representatives $w_{u}$ and $w_{v}$ and is symmetric in $u$ and $v$.

Proof. The relation $w_{u} a w_{v}^{t} \sim e$ is equivalent to $w_{u} \sim w_{v} a$ and to $w_{u} a \sim w_{v}$. Hence the relation is independent of the choice of representatives. By symmetry of $\sim$ (Corollary 5.7), $w_{u} a w_{v}^{t} \sim e \Longrightarrow\left(w_{u} a w_{v}^{t}\right)^{t} \sim e$; hence $w_{v} a w_{u}^{t} \sim e$. Therefore the relation $w_{u} a w_{v}^{t} \sim e$ is symmetric in $u$ and $v$.

Remark 5.9. By the proof of Lemma 5.8 it is evident that every vertex $u$ of $\mathcal{H}$ is the endpoint of exactly $k$ edges, namely $\left\{\left(\left\{u,\left[w_{u} a\right]\right\}, a\right)\right\}_{a \in[k]}$, where $w_{u}$ is any representative of $u$. It follows that $\tau$ is an eigenvector of $\mathcal{H}$ with eigenvalue $k$.

Remark 5.10. By Proposition [5.6, the class of $g(w), w \in \mathcal{F}$, is independent of the choice of $w \in[w]$.

Two Extreme Cases. In [10, Krishnan and Sunder describe two extreme cases: when the action of $[p]$ on $[k]$ is trivial and when the action of $[k]$ on $[p]$ is trivial. In the first case, our definition of $\mathcal{H}$ coincides with Krishnan and Sunder's description of the principal graph, a Cayley-type graph of the subgroup of $S_{p}$ generated by the $[k]$-action. In the second case, it is evident that $\mathcal{H}$ is as trivial as possible; it is the bipartite graph with two vertices and $k$ edges. 


\section{The Action of $G$ on $\mathcal{H}$}

We construct an action of $G$ on $\mathcal{H}$, by which we mean a group homomorphism from $G$ to the root preserving automorphisms of $\mathcal{H}$. The homomorphism we construct is actually injective, but we delay proving this until Section 7

Proposition 6.1. For any $g$ in $G$ and edge $(\{u, v\}, a)$ in $E_{U}$, there exists a unique $b$ in $[k]$ such that for all $w_{u} \in u$ and $w_{v} \in v$, we have

$$
g\left(w_{u} a w_{v}^{t}\right)=g\left(w_{u}\right) b g\left(w_{v}\right)^{t} .
$$

Proof. It is easy to see by induction on length that it suffices to prove the result for a set of generators of $G$. We prove the result for the generating set $\left\{\beta^{-1} \alpha\right\}_{\alpha, \beta \in[p]}$ of $G$.

Fix $g=\beta^{-1} \alpha, \alpha, \beta \in[p]$. We claim the conclusion of the proposition is symmetric in $u$ and $v$. Consider

$$
\begin{aligned}
g\left(w_{u} a w_{v}^{t}\right) & =g\left(w_{u}\right) b g\left(w_{v}\right)^{t} \\
g\left(w_{u} a\right) g\left(w_{v}\right)^{t} & =g\left(w_{u}\right) b g\left(w_{v}\right)^{t} \\
g\left(w_{u} a\right) & =g\left(w_{u}\right) b \\
g\left(w_{v}\right) g\left(w_{u} a\right)^{t} & =g\left(w_{v}\right) b g\left(w_{u}\right)^{t} \\
g\left(w_{v} a w_{u}^{t}\right) & =g\left(w_{v}\right) b g\left(w_{u}\right)^{t}
\end{aligned}
$$

Then (6.1) $\Longleftrightarrow$ (6.2) (by Lemma 5.5) $\Longleftrightarrow$ 6.3) $\Longleftrightarrow$ 6.4 6.5) (by Lemma 5.5), which proves the claim. So we may assume without loss of generality that $u$ is the even vertex.

Let

$$
\left(\gamma_{u}, \epsilon_{u}\right)=w_{u}(\alpha, \beta) \quad \text { and } \quad\left(\gamma_{v}, \epsilon_{v}\right)=w_{v}(\alpha, \beta) \text {. }
$$

By Proposition 5.6 $\gamma_{u}, \epsilon_{u}, \gamma_{v}$, and $\epsilon_{v}$ are independent of the choice of $w_{u}$ and $w_{v}$. Let

$$
x_{u}=g\left(w_{u}\right) \quad \text { and } \quad x_{v}=g\left(w_{v}\right) .
$$

Then

$$
g\left(w_{u} a w_{v}^{t}\right)=x_{u} g\left(w_{v} a\right)^{t}=g\left(w_{u} a\right) x_{v}
$$

by Lemma 5.2 . Hence, there is a $b \in[k]$ such that

$$
g\left(w_{u} a w_{v}^{t}\right)=x_{u} b x_{v} .
$$


Then (using Lemma 5.1 and Lemma 3.4 for the third equality and Lemma 3.4 for the fourth equality)

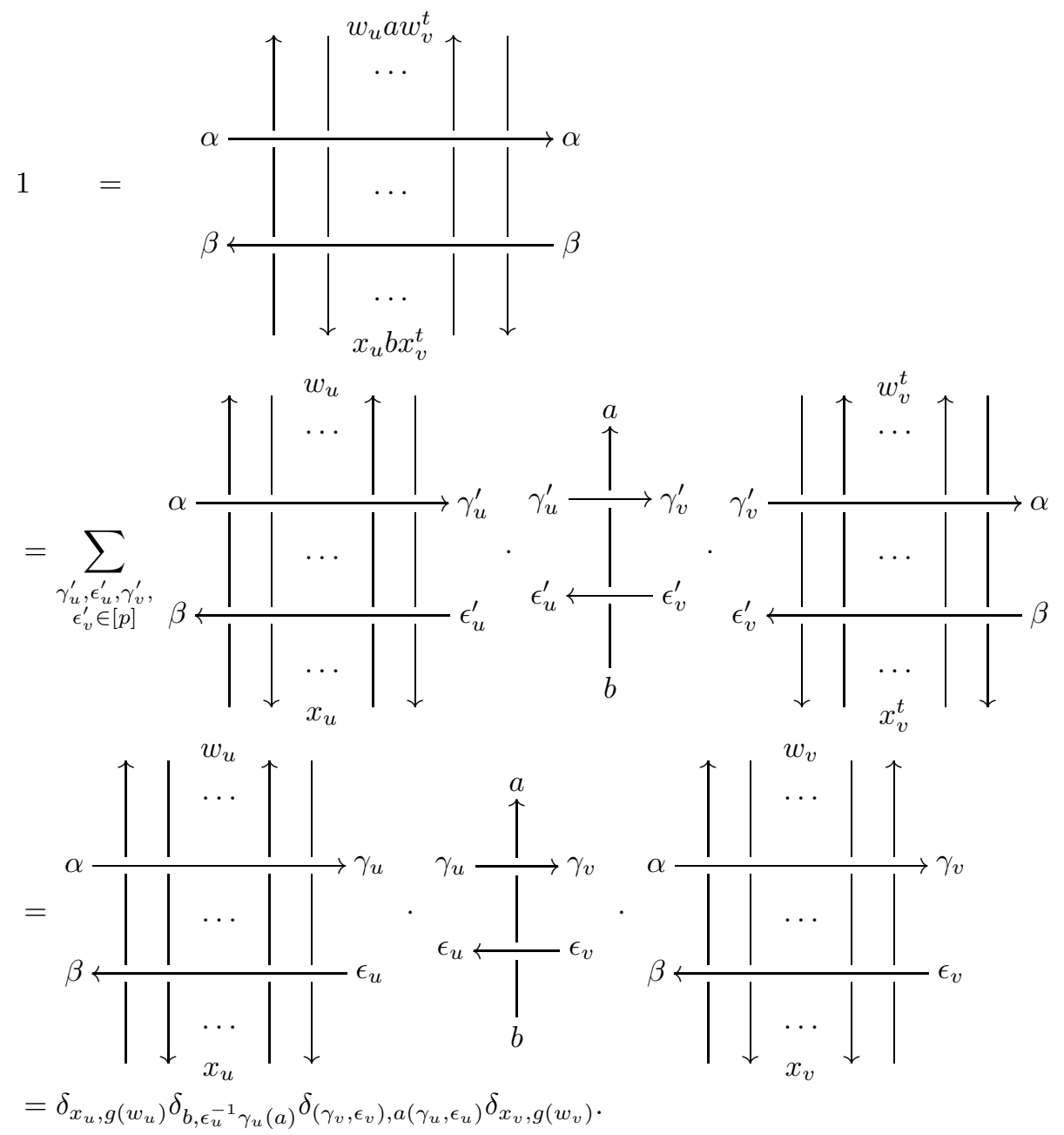

Therefore, $b=\epsilon_{u}^{-1} \gamma_{u}(a)$.

The Group Action. Given any $g \in G,[w] \in V_{U}$, and $(\{u, v\}, a) \in E_{U}$, define $g_{\mathcal{H}}([w])=[g(w)]$ and $g_{\mathcal{H}}((\{u, v\}, a))=\left(\left\{g_{\mathcal{H}}(u), g_{\mathcal{H}}(v)\right\}, b\right)$, where $b$ is as in Proposition 6.1 By Proposition [5.6 it's clear that $[g(w)]$ is independent of the choice of $w \in[w]$. It follows from Proposition 6.1 that $\left(\left\{g_{\mathcal{H}}(u), g_{\mathcal{H}}(v)\right\}, b\right)$ is an edge in $E_{U}$. Therefore $g_{\mathcal{H}}$ is a well-defined graph endomorphism of $\mathcal{H}$. It is evident that $g_{\mathcal{H}}$ preserves the root vertex, and it is an easy exercise to show that $g_{\mathcal{H}} h_{\mathcal{H}}=(g h)_{\mathcal{H}}$ for any $g, h \in G$. Therefore, we have a group homomorphism

$$
\begin{aligned}
G & \rightarrow \operatorname{Aut}(\mathcal{H}) \\
g & \mapsto g_{\mathcal{H}}
\end{aligned}
$$

from $G$ to the group of root-preserving automorphisms of $\mathcal{H}$. When there is no ambiguity, we shall simply write $g$ instead of $g_{\mathcal{H}}$. 


\section{Higher Relative Commutants of $R_{U} \subset R$}

The description of the higher relative commutants given in Corollary 3.6 can be rephrased in terms of the path algebras ([13], [18, [4]) on $\mathcal{H}$ and the action of $G$ on $\mathcal{H}$. We briefly recall some ideas concerning path algebras. Let $V_{\mathcal{H}}^{n}$ denote the complex vector space with orthonormal basis the set of rooted paths of length $n$ on $\mathcal{H}$. If $F \in \operatorname{End}\left(V_{\mathcal{H}}^{n}\right)$, write $F$ in matrix form $\left(F_{\rho_{1}, \rho_{2}}\right)_{\rho_{1}, \rho_{2}}$, where $\rho_{1}$ and $\rho_{2}$ range over the rooted paths of length $n$ on $\mathcal{H}$. The $n$th path algebra $P_{n}$ on $\mathcal{H}$ is the subalgebra of $\operatorname{End}\left(V_{\mathcal{H}}^{n}\right)$,

$$
P_{n}=\left\{F \in \operatorname{End}\left(V_{\mathcal{H}}^{n}\right): F_{\rho_{1}, \rho_{2}}=0 \text {, if } \rho_{1} \text { and } \rho_{2} \text { end at different vertices }\right\}
$$

If for each vertex $v$ of $\mathcal{H}$ we let $X_{v}^{n} \subset V_{\mathcal{H}}^{n}$ denote the space spanned by paths ending at $v$, then

$$
P_{n}=\bigoplus_{v \in \mathcal{H}} \operatorname{End}\left(X_{v}^{n}\right)
$$

Note that $X_{v}$ may be 0 .

Since $F$ stabilizes the end vertices of paths, there is a natural inclusion map $i: P_{n} \rightarrow P_{n+1}$, where $i(F)$ preserves the part of a path after its $n$th vertex. More precisely, if

$$
\begin{gathered}
\mu_{1}=\rho_{1} \circ \nu_{1}, \\
\mu_{2}=\rho_{2} \circ \nu_{2}
\end{gathered}
$$

where $\rho_{i}$ has length $n$ and $\nu_{i}$ has length $1, i=1,2$, then

$$
i(F)_{\mu_{1}, \mu_{2}}=\delta_{\nu_{1}, \nu_{2}} F_{\rho_{1}, \rho_{2}} .
$$

Thus we have a tower of finite dimensional $C^{*}$-algebras

$$
\mathbb{C}=P_{0} \subset P_{1} \subset \ldots P_{n} \subset \ldots
$$

Let $\operatorname{tr}_{n}$ be the trace on $P_{n}$ such that the trace of a minimal projection in $\operatorname{End}\left(X_{v}^{n}\right)$ is $\frac{1}{k^{n}}$ times the weight of $\tau$ on $v$ (which is 1 for all $v$ ). Since $\tau$ is a $k$-eigenvector, the $\operatorname{traces} \operatorname{tr}_{n}$ are compatible with the inclusion maps, and we obtain a trace tr on $\bigcup_{n} P_{n}$ with $\operatorname{tr}(1)=1$.

If $\rho$ is a rooted path of length $n$, let $\rho(i)$ denote the $i$ th edge of $\rho$. Define $E_{n-1} \in P_{n}, n>1$, by

$$
\left(E_{n-1}\right)_{\rho_{1}, \rho_{2}}=\frac{1}{k} \delta_{\rho_{1}(n-1), \rho_{1}(n)} \delta_{\rho_{2}(n-1), \rho_{2}(n)}
$$

if $\rho_{1}$ and $\rho_{2}$ end at the same vertex. Then $\left(E_{n}\right)_{n}$ is a sequence of Jones projections with modulus $k$, and tr satisfies the Markov property with respect to $\left(E_{n}\right)_{n}$. We can sum this up by saying that $\mathcal{H}$ with weight vector $\tau$ is the principal graph of the tower $\left(P_{n}\right)_{n}$.

There is a natural group action of $G$ on $V_{\mathcal{H}}^{n}$ for each $n$, arising from the natural permutation action of $\operatorname{Aut}(\mathcal{H})$ on the set of rooted paths of length $n$. Namely, if $g \in \operatorname{Aut}(\mathcal{H})$ and $v_{0} e_{1} v_{1} \ldots e_{n} v_{n}$ is a rooted path, then

$$
g\left(v_{0} e_{1} v_{1} \ldots e_{n} v_{n}\right)=g\left(v_{0}\right) g\left(e_{1}\right) g\left(v_{1}\right) \ldots g\left(e_{n}\right) g\left(v_{n}\right) .
$$

For each $n$ let $B_{n}$ be the subalgebra of $P_{n}$ that commutes with the action of $G$. It is easy to check that $B_{n} \subset B_{n+1}$ for all $n$. 
Theorem 7.1. There is a trace-preserving $*$-isomorphism from the tower of higher relative commutants of $R_{U} \subset R$ to the tower of algebras $\left(B_{n}\right)_{n}$. The isomorphism preserves Jones projections.

The rest of this section is devoted to proving the theorem. By Remark [5.9] if $a_{1} a_{2} \ldots a_{n} \in \mathcal{F}$, there is a unique rooted path $v_{0} e_{1} v_{1} \ldots e_{n} v_{n}$ on $\mathcal{H}$ such that $e_{i}=\left(\left\{v_{i-1}, v_{i}\right\}, a_{i}\right), 1 \leq i \leq n$. Denote this path $\phi\left(a_{1} \ldots a_{n}\right)$. It is easy to see that

$$
\begin{aligned}
\phi: \mathcal{F} & \rightarrow\{\text { Rooted paths on } \mathcal{H}\} \\
w & \mapsto \phi(w)
\end{aligned}
$$

is a bijection of sets. Thus $\phi$ extends to a unitary vector space isomorphism of $V^{n}$ with $V_{\mathcal{H}}^{n}$, which we also denote by $\phi$.

Lemma 7.2. If $\phi\left(a_{1} a_{2} \ldots a_{n}\right)=v_{0} e_{1} v_{1} \ldots e_{n} v_{n}$, then $v_{i}=\left[a_{1} \ldots a_{i}\right], 1 \leq i \leq n$.

Proof. We prove the lemma by induction. Since $v_{0}=[e]$, it suffices to show that if $v_{i-1}=[w]$ then $v_{i}=\left[w a_{i}\right], 1 \leq i \leq n$. This follows from the fact that $e_{i}=$ $\left(\left\{v_{i-1}, v_{i}\right\}, a_{i}\right)$ is an edge.

The lemma immediately implies the following corollary.

Corollary 7.3. If $w \in \mathcal{F}$, then $w \sim e$ if and only if $\phi(w)$ is a closed path.

Proposition 7.4. If $g \in G$ and $w \in \mathcal{F}$, then

$$
\phi(g(w))=g(\phi(w)) .
$$

Proof. Let $w=a_{1} \ldots a_{n}$, where $a_{i} \in[k]$ for $1 \leq i \leq n$. Let $\phi(w)=* e_{1} v_{1} \ldots e_{n} v_{n}$, where $*$ denotes the root. We prove the result by induction on $n$. The result is trivial when $n=0$. Let $w^{\prime}=a_{1} \ldots a_{n-1}$. Clearly $\phi\left(w^{\prime}\right)=* e_{1} v_{1} \ldots e_{n-1} v_{n-1}$. By the induction hypothesis, we may assume that $g\left(\phi\left(w^{\prime}\right)\right)=\phi\left(g\left(w^{\prime}\right)\right)$. Thus, there exist $b_{i}, u_{i}, f_{i}, 1 \leq i \leq n-1$, such that $g\left(w^{\prime}\right)=b_{1} \ldots b_{n-1}$ and $g\left(* e_{1} v_{1} \ldots e_{n-1} v_{n-1}\right)=$ $* f_{1} u_{1} \ldots f_{n-1} u_{n-1}$ where $f_{i}=\left(\left\{u_{i-1}, u_{i}\right\}, b_{i}\right)$ for each $i$. By Lemma $7.2 u_{n-1}=$ $\left[w^{\prime}\right]$. By definition, $g\left(e_{n}\right)$ is the edge $\left(\left\{\left[g\left(w^{\prime}\right)\right],[g(w)]\right\}, b\right)$ such that $g\left(w^{\prime} a_{n} w^{t}\right)=$ $g\left(w^{\prime}\right) b g(w)^{t}$. So we have

$$
\begin{aligned}
g\left(w^{\prime} a_{n} w^{t}\right) & =g\left(w w^{t}\right) \\
& =g(w) g(w)^{t} \quad \text { (Lemma 5.5). }
\end{aligned}
$$

Therefore, $g(w)=g\left(w^{\prime}\right) b$. So we have

$$
\begin{aligned}
g(\phi(w)) & =g\left(\phi\left(w^{\prime}\right)\right) \circ g\left(v_{n-1} e_{n} v_{n}\right) \\
& =\phi\left(g\left(w^{\prime}\right)\right) \circ g\left(v_{n-1} e_{n} v_{n}\right) \\
& =\phi\left(g\left(w^{\prime}\right) b\right) \\
& =\phi(g(w)) .
\end{aligned}
$$

Since $\phi$ is an isomorphism, the previous proposition implies the following corollary.

Corollary 7.5. The homomorphism $G \rightarrow \operatorname{Aut}(\mathcal{H})$ is injective. 
Proof of Theorem 7.1. Suppose $F \in \operatorname{End}\left(V^{n}\right)$. Then $\phi F \phi^{-1} \in \operatorname{End}\left(V_{\mathcal{H}}^{n}\right)$, and if $w_{1}, w_{2} \in \mathcal{F}$ are of length $n$, then

$$
F_{w_{1} w_{2}^{t}}=\left(\phi F \phi^{-1}\right)_{\phi\left(w_{1}\right), \phi\left(w_{2}\right)} .
$$

We first prove that the injective $*$-homomorphism

$$
\begin{aligned}
\operatorname{End}\left(V^{n}\right) & \rightarrow \operatorname{End}\left(V_{\mathcal{H}}^{n}\right) \\
F & \mapsto \phi F \phi^{-1}
\end{aligned}
$$

maps $\widetilde{B}_{n}$ onto $B_{n}$. We claim that $F$ satisfies the first condition of Corollary 3.6 iff $\phi F \phi^{-1} \in P_{n}$. If $w_{1}, w_{2}$ are as above, then $w_{1} w_{2}^{t} \sim e \Longleftrightarrow w_{1} \sim w_{2} \Longleftrightarrow \phi\left(w_{1}\right)$ and $\left.\phi_{(} w_{2}\right)$ end at the same vertex (by Lemma 7.2). Since $w \sim e$ is equivalent to $w$ satisfying the first condition of Corollary 3.6. the claim follows.

Now assume that $F$ satisfies the first condition of Corollary 3.6. Then we claim that $F$ satisfies the second condition of the corollary iff $\phi F \phi^{-1}$ commutes with the $G$-action. Note that $F^{\prime} \in P_{n}$ commutes with the $G$-action iff $F_{\rho_{1}, \rho_{2}}^{\prime}=F_{g\left(\rho_{1}\right), g\left(\rho_{2}\right)}^{\prime}$ for all rooted paths $\rho_{1}, \rho_{2}$ of length $n$ and $g \in G$. Hence it suffices to prove that if $w_{1}, w_{2} \in \mathcal{F}$ are of length $n$ and $g \in G$, then

$$
F_{g\left(w_{1} w_{2}^{t}\right)}=\left(\phi F \phi^{-1}\right)_{g \phi\left(w_{1}\right), g \phi\left(w_{2}\right)^{t}} .
$$

Note that $w_{1} w_{2}^{t} \sim e \Longleftrightarrow g\left(w_{1} w_{2}^{t}\right) \sim e$, and that $\phi\left(w_{1}\right)$ and $\phi\left(w_{2}\right)$ end at the same vertex iff $g\left(\phi\left(w_{1}\right)\right)$ and $g\left(\phi\left(w_{2}\right)\right)$ end at the same vertex. Thus, if $w_{1} \nsim w_{2}$, then both sides of (7.2) are 0 , since $F$ satisfies the first condition of Corollary 3.6, So assume $w_{1} \sim w_{2}$. Then (7.2) follows from Proposition 7.4. Lemma 5.5, and (7.1). It follows from the two claims that $\phi \widetilde{B}_{n} \phi^{-1}=B_{n}$.

It is easy to see that the isomorphism of $\widetilde{B}_{n}$ with $B_{n}$ implemented by $\phi$ respects the inclusion maps, preserves the trace, and maps $\widetilde{E}_{n}$ to $E_{n}$, for each $n$. The proof then follows from Theorem 2.1.

\section{Principal Graph of $R_{U} \subset R$}

For each vertex $v \in \mathcal{H}$, let $G_{v}$ be the subgroup of $G$ that fixes the vertex $v$. Consider the action of $G$ on the vertex set of $\mathcal{H}$, and for each $G$-orbit fix a representative vertex $w$. Let $O$ denote the set of representative vertices. Denote the orbit of $w$ by $o(w)$. For every $w \in O$ and $u \in o(w)$, fix $g_{u} \in G$ satisfying $g_{u}(u)=w$. For any group $H$, let $\hat{H}$ denote the finite dimensional irreducible representations of $H$. Each $g_{u}$ induces the isomorphism $\operatorname{Ad}_{g_{u}}^{*}: \hat{G_{w}} \rightarrow \hat{G_{u}}$, which we denote by $\hat{g_{u}}$,

$$
\hat{g_{u}}: \hat{G_{w}} \rightarrow \hat{G_{u}} .
$$

If $u$ and $w$ are vertices of $\mathcal{H}$, let $E_{u, w}$ be the vector space with basis the set of edges between $u$ and $w . E_{u, w}$ is naturally a $\left(G_{u} \cap G_{w}\right)$-module.

Definition 8.1. Define the graphs $\Gamma^{0}$ and $\Gamma$ as follows. The vertex set of $\Gamma^{0}$ is $\amalg_{w \in O} \hat{G_{w}}$, the disjoint union of the sets $\hat{G_{w}}$. Let $\lambda_{v} \in \hat{G_{v}}$ and $\lambda_{w} \in \hat{G_{w}}$, for $v, w \in O$, be vertices of $\Gamma^{0}$. Consider the action of $G_{w}$ on $o(v)$, and let $S$ be a set of representatives of the $G_{w}$ orbits. The number of edges between $\lambda_{w}$ and $\lambda_{v}$ is

$$
\sum_{u \in S} \operatorname{dim}\left(\operatorname{Hom}\left(\lambda_{w}, \operatorname{Ind}_{G_{u} \cap G_{w}}^{G_{w}}\left(\left(\operatorname{Res}_{G_{u} \cap G_{w}}^{G_{u}} \hat{g_{u}}\left(\lambda_{v}\right)\right) \otimes E_{u, w}\right)\right)\right) .
$$


Let $\tau_{\Gamma}$ be the vector of weights on $\Gamma^{0}$ given by $\lambda_{w} \mapsto \operatorname{dim} \lambda_{w} \cdot c$, where if $\lambda_{w} \in \hat{G_{w}}$ then $c$ is the order of $o(w)$. Let $1_{*}$ denote the trivial representation of $G_{*}$. Define $\Gamma$ to be the connected component of $\Gamma^{0}$ containing the vertex $1_{*}$.

A simple calculation involving Frobenious reciprocity shows that 8.1) is symmetric in $\lambda_{v}$ and $\lambda_{w}$, so $\Gamma^{0}$ and $\Gamma$ are well-defined.

Theorem 8.2. The graph $\Gamma$ with weight vector $\tau_{\Gamma}$ and distinguished vertex $1_{*}$ is the principal graph of $R_{U} \subset R$.

The proof follows Lemma 8.7 The following remark applies to examples discussed later.

Remark 8.3. Suppose the following assumptions hold:

(1) For every vertex $w \in \mathcal{H}$ and every $G$-orbit $o \subset\{$ vertices of $\mathcal{H}\}, G_{w}$ acts transitively on $\{u \in o: u$ is a neighbor of $w\}$.

(2) $\mathcal{H}$ does not have multiple edges (distinct edges of $\mathcal{H}$ have distinct endpoint sets).

Then the edge formula (8.1) simplifies to

$$
\operatorname{dim}\left(\operatorname{Hom}\left(\lambda_{w}, \operatorname{Ind}_{G_{u} \cap G_{w}}^{G_{w}}\left(\operatorname{Res}_{G_{u} \cap G_{w}}^{G_{u}} \hat{g_{u}}\left(\lambda_{v}\right)\right)\right)\right)
$$

where $u$ is any vertex in $o(v)$ that neighbors $w$. If such an edge does not exist, then there are no edges between $\lambda_{v}$ and $\lambda_{w}$.

Remark 8.4. It is easy to see that our description of the principal graph coincides with Krishnan and Sunder's in the two extreme cases discussed at the end of Section 5 If $G$ is trivial, then $\Gamma$ is identical to $\mathcal{H}$. On the other hand, suppose $\mathcal{H}$ is the bipartite graph with two vertices $\{0,1\}$ and $k$ edges. Note that $G$ fixes both vertices. Hence, $\Gamma^{0}$ is the connected graph whose vertices are the finite dimensional irreducible $G$-representations and where the number of edges between two vertices, $\lambda_{1}$ and $\lambda_{2}$, is the multiplicity of $\lambda_{1}$ in $\lambda_{2} \otimes E_{0,1}$. Then $\Gamma$ is the connected component containing the trivial representation as a vertex.

By Theorem 7.1 the principal graph of $R_{U} \subset R$ is calculated from the tower $\left(B_{n}\right)_{n}$. For each nonnegative integer $n$ and vertex $v \in \mathcal{H}$, let $p_{v}^{n} \in \operatorname{End}\left(V_{\mathcal{H}}^{n}\right)$ be the orthogonal projection to the span of paths ending at $v$. Recall that $G$ acts on $V_{\mathcal{H}}^{n}$ by its action on paths of length $n$. Let $C_{n}$ be the subalgebra of $\operatorname{End}\left(V_{\mathcal{H}}^{n}\right)$ generated by $\left\{p_{v}^{n}\right\}_{v \in \mathcal{H}}$ and the $G$-action on $V_{\mathcal{H}}^{n}$. Then, by definition,

$$
B_{n}=C_{n}^{\prime} \cap \operatorname{End}\left(V_{\mathcal{H}}^{n}\right) \text {. }
$$

Thus, the simple summands of $B_{n}$ are in bijection with the irreducible $C_{n}$-modules in $V_{\mathcal{H}}^{n}$. Let $\mathcal{M}_{n}$ be the set of isomorphism classes of irreducible $C_{n}$-modules contained in $V_{\mathcal{H}}^{n}$. Note that any $C_{n}$-module is naturally a $G$-module.

For each $w \in O$,

$$
q_{w}=\sum_{u \in o(w)} p_{u}^{n}
$$

is clearly a central (possibly 0) projection in $C_{n}$, and $\left\{q_{w}\right\}_{w \in O}$ is a partition of unity. Hence, for any $M \in \mathcal{M}_{n}$, there is a unique $w \in O$ such that $q_{w} M \neq 0$, or equivalently, $q_{w} M=M$. Let $\mathcal{M}_{w, n}$ denote the subset

$$
\mathcal{M}_{w, n}=\left\{M \in \mathcal{M}_{n}: q_{w} M=M\right\} .
$$


So $\mathcal{M}_{n}=\amalg_{w \in O} \mathcal{M}_{w, n}$. Also, let $\Lambda_{w, n} \subset \hat{G_{w}}$ be the set of irreducible $G_{w}$ representations contained in $p_{w}^{n} V_{\mathcal{H}}^{n}$.

Lemma 8.5. For each nonnegative integer $n$ and $w \in O$, the map

$$
\begin{gathered}
\mathcal{M}_{w, n} \longleftrightarrow \Lambda_{w, n} \\
M \longmapsto p_{w}^{n} M
\end{gathered}
$$

is a bijection and $\operatorname{dim}(M)=\operatorname{dim}\left(p_{w}^{n} M\right) \cdot c$, where $c$ is the order of $o(w)$.

Proof. Since $n$ is fixed, let $p_{v}=p_{v}^{n}$, for each $v \in \mathcal{H}$. Recall the elements $g_{u} \in G, u \in$ $o(w)$, defined in the discussion before Definition 8.1. Clearly $\left\{p_{v} g_{v}^{-1} g_{u} p_{u}\right\}_{v, u \in o(w)}$ is a set of matrix units for the partition $\left\{p_{u}\right\}_{u \in o(w)}$ of $q_{w}$. Let $V_{w}$ be the complex vector space with orthonormal basis indexed by the $G$-orbit of $w$. The matrix units give rise to isomorphisms

$$
\begin{aligned}
q_{w} C_{n} & \longleftrightarrow \operatorname{End}\left(V_{w}\right) \otimes p_{w} C_{n} p_{w} \\
q_{w} V_{\mathcal{H}}^{n} & \longleftrightarrow V_{w} \otimes p_{w} V_{\mathcal{H}}^{n}
\end{aligned}
$$

that intertwine the action of $q_{w} C_{n}$ on $q_{w} V_{\mathcal{H}}^{n}$ and the action of $\operatorname{End}\left(V_{w}\right) \otimes p_{w} C_{n} p_{w}$ on $V_{w} \otimes p_{w} V_{\mathcal{H}}^{n}$. Thus, a subspace $M \subset q_{w} V_{\mathcal{H}}^{n}$ is an irreducible $C_{n}$-module iff $p_{w} M$ is an irreducible $p_{w} C_{n} p_{w}$-module. The dimension formula also follows. But it is easy to see that $p_{w} C_{n} p_{w}=p_{w}(\mathbb{C} G) p_{w}=\mathbb{C} G_{w}$. Therefore, the isomorphism class of $p_{w} M$ as a $p_{w} C_{n} p_{w}$-module is determined by its isomorphism class as a $G_{w}$-representation. So the map $M \mapsto p_{w} M$ is an injection from $\mathcal{M}_{w, n}$ to $\Lambda_{w, n}$. By the definition of $\mathcal{M}_{w, n}$ and $\Lambda_{w, n}$, the map is obviously surjective.

Recall the natural isomorphism $V^{n} \cong\left(V^{1}\right)^{\otimes n}$. Using the isomorphisms $\phi$ : $V^{n} \rightarrow V_{\mathcal{H}}^{n}$, we obtain an isomorphism

$$
i: V_{\mathcal{H}}^{n} \rightarrow\left(V_{\mathcal{H}}^{1}\right)^{\otimes n} .
$$

By abuse of notation we also denote any isomorphism of the form $V_{\mathcal{H}}^{n+m} \cong V_{\mathcal{H}}^{n} \otimes V_{\mathcal{H}}^{m}$ that arises from $\phi$ by $i$.

Consider the Bratteli diagram of $B_{n} \subset B_{n+1}$. By (8.3) we may identify its vertices with $\mathcal{M}_{n} \cup \mathcal{M}_{n+1}$. Note that if $M \in \mathcal{M}_{n}$ and $M^{\prime} \in \mathcal{M}_{n+1}$, the number of edges between them is $\operatorname{dim}\left(\operatorname{Hom}\left(M^{\prime}, i^{-1}\left(M \otimes V_{\mathcal{H}}^{1}\right)\right)\right)$.

Lemma 8.6. Fix $n$, a nonnegative integer, and $v \in O$. Take any $\lambda_{v} \in \Lambda_{v, n}$. Let $M \in \mathcal{M}_{v, n}$ be the irreducible $C_{n}$-module corresponding to $\lambda_{v}$ via the bijection in Lemma 8.5. Fix $w \in O$, and let $S$ be as in Definition 8.1. Then there is an isomorphism of $G_{w}$-representations

$$
p_{w}^{n+1}\left(i^{-1}\left(M \otimes V_{\mathcal{H}}^{1}\right)\right) \cong \bigoplus_{u \in S} \operatorname{Ind}_{G_{u} \cap G_{w}}^{G_{w}}\left(\left(\operatorname{Res}_{G_{u} \cap G_{w}}^{G_{u}} \hat{g_{u}}\left(\lambda_{v}\right)\right) \otimes E_{u, w}\right) .
$$

Proof. By definition,

$$
\begin{aligned}
p_{w}^{n+1}\left(i^{-1}\left(M \otimes V_{\mathcal{H}}^{1}\right)\right) & =\bigoplus_{u \in o(v)}\left(p_{u}^{n} M\right) \otimes E_{u, w} \\
& =\bigoplus_{u \in S} G_{w}\left(\left(p_{u}^{n} M\right) \otimes E_{u, w}\right)
\end{aligned}
$$

as vector spaces. As $G_{w}$-representations,

$$
G_{w}\left(\left(p_{u}^{n} M\right) \otimes E_{u, w}\right) \cong \operatorname{Ind}_{G_{u} \cap G_{w}}^{G_{w}}\left(\left(\operatorname{Res}_{G_{u} \cap G_{w}}^{G_{u}} p_{u}^{n} M\right) \otimes E_{u, w}\right)
$$


because $G_{u} \cap G_{w}$ is the stabilizer subgroup of $\left(p_{u}^{n} M\right) \otimes E_{u, w}$ in the left-hand side of (8.8). Finally,

$$
\left(p_{u}^{n} M\right)=\hat{g_{u}}\left(p_{v}^{n} M\right) \cong \hat{g_{u}}\left(\lambda_{v}\right) ;
$$

so the lemma follows from (8.7) and (8.8).

Lemma 8.7. Let $M \in \mathcal{M}_{n}$ and $M^{\prime}=E_{n+1}\left(i^{-1}\left(M \otimes V_{\mathcal{H}}^{2}\right)\right)$, where $E_{n+1}$ is the Jones projection. Then $M^{\prime} \in \mathcal{M}_{n+2}$ and $M \leftrightarrow \lambda \Longrightarrow M^{\prime} \leftrightarrow \lambda$, where $\leftrightarrow$ denotes the bijection in Lemma 8.5 .

Proof. Let $M \in \mathcal{M}_{v, n}, v \in O$. Let $S$ be the set of rooted paths of length two on $\mathcal{H}$ that have the form

$$
* e v e *
$$

for some edge $e$ and vertex $v$. Note that $G_{*}$ fixes $\sum_{\rho \in S} \rho$. Then

$$
\begin{aligned}
p_{v}^{n+2} E_{n+1}\left(i^{-1}\left(M \otimes V_{\mathcal{H}}^{2}\right)\right) & =E_{n+1}\left(i^{-1}\left(\left(p_{v}^{n} M\right) \otimes V_{\mathcal{H}}^{2}\right)\right) \\
& =i^{-1}\left(\left(p_{v}^{n} M\right) \otimes E_{1}\left(V_{\mathcal{H}}^{2}\right)\right) \\
& =i^{-1}\left(p_{v}^{n} M \otimes \mathbb{C}\left(\sum_{\rho \in S} \rho\right)\right),
\end{aligned}
$$

which is equivalent to $p_{v}^{n} M \otimes G_{v} 1$, as $G_{v}$-representations. Therefore, $p_{v}^{n} M \cong$ $p_{v}^{n+2} M^{\prime}$ as $G_{v}$-representations, which proves the result.

Proof of Theorem 8.2. By Theorem 7.1 we can calculate the principal graph of $R_{U} \subset R$ from the tower $\left(B_{n}\right)_{n}$. By Lemma 8.5 and the discussion preceding it, we have an injection for each $n$ from the vertices of the Bratteli diagram of $B_{n} \subset B_{n+1}$ to the vertex set of $\Gamma^{0}$. As previously discussed, we identify the vertex set of this Bratteli diagram with $\mathcal{M}_{n} \cup \mathcal{M}_{n+1}$. $\mathcal{M}_{n}$ maps onto $\amalg_{o \in O} \Lambda_{o, n}$, and Lemma 8.6 implies that $\mathcal{M}_{n+1}$ maps onto all the vertices of $\Gamma^{0}$ that neighbor at least one vertex in $\amalg_{o \in O} \Lambda_{o, n}$. It also implies that this injection preserves the multiplicity of edges between pairs of vertices. Therefore, the injection extends to an isomorphism of the Bratteli diagram of $B_{n} \subset B_{n+1}$ with the subgraph of $\Gamma^{0}$ connected to $\amalg_{o \in O} \Lambda_{o, n}$. Note that the sole element of $\mathcal{M}_{0}$ is $V_{\mathcal{H}}^{0}$, which corresponds to the trivial $G_{*}$-representation. It follows that $\Gamma$ is the principal graph of $R_{U} \subset R$ and $1_{*}$ is the distinguished vertex. If $M \in \mathcal{M}_{n, w}$, then the trace weight on $M$ in the Bratteli diagram of $B_{n} \subset B_{n+1}$ is $\operatorname{dim}(M)$ divided by the normalization factor of $k^{n}$. Hence, the trace weight on $M$ in $\Gamma$ is $\operatorname{dim}(M)=c \cdot \operatorname{dim}\left(p_{w}^{n} M\right)$, where $c$ is the order of $o(w)$ (Lemma 8.5). Finally, Lemma 8.7 ensures that the injection from simple summands of $B_{n}$ to simple summands of $B_{n+2}$ implemented by the Jones projection (the reflection property of the Bratteli diagram) is consistent with the injection induced by the graph $\Gamma$.

Corollary 8.8. The subfactor $R_{U} \subset R$ has infinite depth iff its graph $\mathcal{H}$ is infinite.

Proof. If $\mathcal{H}$ is finite, then $O$ is a finite set and for each $v \in O, G_{v}$ is a finite group, hence $\hat{G}_{v}$ is finite. So $\Gamma$ is finite.

On the other hand, assume $\mathcal{H}$ is infinite. Then we claim that for every nonnegative integer $n$ there exists a vertex whose minimum distance from the root of $\mathcal{H}$ is $n$. For since every vertex of $\mathcal{H}$ is adjacent to exactly $k$ edges (Remark 5.9), 
there cannot be more than $k^{n}$ vertices whose distance from the root is $n$. Fix any nonnegative integer $n$ and let

$$
\rho=* e_{1} v_{1} e_{2} v_{2} \ldots e_{n} v_{n}
$$

be a path of minimal length from $*$ to $v_{n}$. Let $v_{0}=*$. Then for each $i, 0 \leq i \leq n$, the minimal distance from $v_{i}$ to $*$ is $i$. Since distance to the root is preserved by the action of $G$, the vertices $v_{i}$ are in pairwise distinct $G$-orbits. So we may choose $O$ such that $v_{i} \in O, 0 \leq i \leq n$. Then the trivial representation of $G_{v_{i}}$, denoted $1_{i}$, is a vertex of $\Gamma^{0}$ for $0 \leq i \leq n$. Moreover, for each $i, 0 \leq i \leq n-1$, the vertices $1_{i}$ and $1_{i+1}$ are neighbors in $\Gamma^{0}$. Therefore, $1_{i}$ is a vertex of $\Gamma$ for $0 \leq i \leq n$; hence $\Gamma$ contains at least $n$ vertices. Since $n$ was arbitrary, $\Gamma$ must be infinite.

\section{Tree Symmetry in Krishnan-Sunder Subfactors}

Let $F G$ be the free group on the alphabet $[k]$ modulo the relation $a^{-1}=a$ for all $a \in[k]$. Consider the natural map $\mathcal{F} \rightarrow F G$. Given a word $w \in \mathcal{F}$, define its reduced form $w_{\text {red }}$ to be the shortest word in $\mathcal{F}$ with the same image in $F G$ as $w$. We may obtain $w_{\text {red }}$ from $w$ by repeatedly canceling double letters in $w$.

We now construct a graph $\mathcal{T}_{k}=\left(V_{\mathcal{T}}, E_{\mathcal{T}}\right)$. Let $V_{\mathcal{T}}$ be the set of reduced words in $\mathcal{F}$ and let

$$
E_{\mathcal{T}}=\left\{(u, v, a): u, v \in V_{\mathcal{T}}, a \in[k], \text { and } u a=v\right\} .
$$

Define $\mathcal{T}_{k}$ to be the rooted graph $\left(V_{\mathcal{T}}, E_{\mathcal{T}}\right)$, in which the endpoints of $(u, v, a)$ are $u$ and $v$, and the root vertex is the empty word. It is evident that $\mathcal{T}_{k}$ is a tree. (Let $a_{1} \ldots a_{n}$ be any word of length $n$ on $[k]$ without repetitions. Then $a_{1} \ldots a_{n}$, as a vertex of $\mathcal{T}_{k}$, is in the $n$th generation of $\mathcal{T}_{k}$ and its parent vertex is $a_{1} \ldots a_{n-1}$.) See Figure 2 for a sketch of the tree $\mathcal{T}_{k}$ in which each edge $(u, v, a)$ is labeled by $a$. Clearly each vertex of $\mathcal{T}_{k}$ is the endpoint of exactly $k$ edges labeled $1,2, \ldots, k$.

Define the graph morphism $\pi: \mathcal{T}_{k} \rightarrow \mathcal{H}$ as follows:

$$
\begin{aligned}
\pi: \mathcal{T}_{k} & \rightarrow \mathcal{H} \\
u & \in V \mapsto[u] \in V_{\mathcal{H}} \\
(u, v, a) & \in E \mapsto(\{[u],[v]\}, a) \in E_{\mathcal{H}} .
\end{aligned}
$$

It is easy to check that $\pi$ is well-defined. We will show that $\pi$ is the universal covering map of $\mathcal{H}$ and gives rise to "tree symmetries" of Krishnan-Sunder subfactors.

We construct a tower of tree algebras from $\mathcal{T}_{k}$ in a fashion analogous to the construction of $\left(B_{n}\right)_{n}$. Let

$$
\mathbb{C}=Q_{0} \subset Q_{1} \subset \ldots Q_{n} \subset \ldots
$$

be the sequence of path algebras on the graph $\mathcal{T}_{k}$ (see the beginning of Section 7 ) with the trace given by a constant weight vector on $\mathcal{T}_{k}$. Let $V_{\mathcal{T}}^{n}$ denote the space on which $Q_{n}$ acts, that is, the complex vector space with orthonormal basis the set of rooted paths of length $n$ on $\mathcal{T}_{k}$. Denote the group of root-preserving automorphisms of $\mathcal{T}_{k}$ by $\operatorname{Aut}\left(\mathcal{T}_{k}\right)$. There is a natural action of $\operatorname{Aut}\left(\mathcal{T}_{k}\right)$ on the set of rooted paths of length $n$, and hence on $V_{\mathcal{T}}^{n}$. For each $n$ let $A_{n}(k) \subset Q_{n}$ be the subalgebra commuting with the action of $\operatorname{Aut}\left(\mathcal{T}_{k}\right)$. Then $\left(A_{n}(k)\right)_{n}$ is an increasing sequence of algebras with a faithful trace inherited from $\left(Q_{n}\right)_{n}$. Note that $\left(A_{n}(k)\right)_{n}$ depends only on $k$, not on the biunitary $U$. 


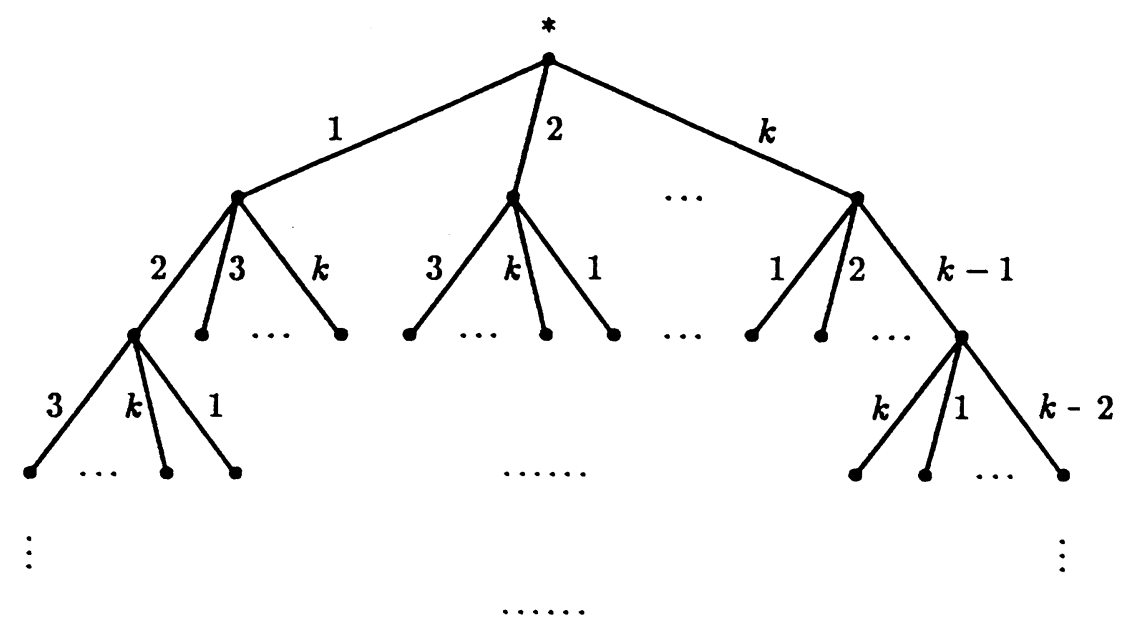

FiguRE 2. $\mathcal{T}_{k}$

Theorem 9.1. Let $U \in M_{p}(\mathbb{C}) \otimes M_{k}(\mathbb{C})$ be a permutation biunitary and let $\left(B_{n}\right)_{n}$ be as defined in Section 7 . There is a canonical injective *-morphism of algebras $\iota: \bigcup_{n} A_{n}(k) \rightarrow \bigcup_{n} B_{n}$ such that $\iota\left(A_{n}(k)\right) \subset B_{n}$ for each $n$ and $\iota$ preserves the trace and the Jones projections.

The proof appears after Corollary 9.5 .

\section{Proposition 9.2.}

$$
\pi^{r}: \mathcal{T}_{k}^{r} \rightarrow \mathcal{H}^{r}
$$

is the universal covering map of $\mathcal{H}^{r}$ ( $\pi^{r}$ is defined in Section 4$)$.

Proof. We will prove that $\pi$ is a local isomorphism. The proof then follows from Lemma 4.1 ( $\pi$ is obviously surjective) and the fact that any tree is contractible (see, for example, [1]).

If $u \in V_{\mathcal{T}}$, then the set of edges in $\mathcal{T}$ which have $u$ as an endpoint is $E_{\mathcal{T}}(u)=$ $\{(u, u a, a): a \in[k]\}$. The set of edges in $\mathcal{H}$ which have $[u]$ as an endpoint is

$$
E_{\mathcal{H}}([u])=\{(\{[u],[u a]\}, a): a \in[k]\} .
$$

Hence,

$$
\left.\pi^{r}\right|_{E_{\mathcal{T}}(u)}: E_{\mathcal{T}}(u) \rightarrow E_{\mathcal{H}}([u])
$$

is a bijection for each $u$.

Corollary 9.3. The map $\pi_{*}$, induced by $\pi$, from the rooted paths of length $n$ on $\mathcal{T}_{k}$ to the rooted paths of length $n$ on $\mathcal{H}$ is a bijection for each $n$.

Proof. This follows from the theory of covering maps (see, for example, [1]).

Remark 9.4. Let $w=a_{1} a_{2} \ldots a_{n}$ be a word in $\mathcal{F}$ and let $\phi(w)$ be the rooted path on $\mathcal{H}$ associated to $w$ (Section 7). Then

$$
\pi_{*}^{-1}(\phi(w))=v_{0} e_{1} v_{1} e_{2} \ldots e_{n} v_{n}
$$

where $v_{0}$ is the empty word and $e_{i}=\left(v_{i-1}, v_{i}, a_{i}\right)$. We leave it to the reader to check that $v_{i}$ is the reduced form of $a_{1} a_{2} \ldots a_{i}$ for each $i>1$. 
Corollary 9.5. There is an injective group homomorphism

$$
\pi^{*}: \operatorname{Aut}(\mathcal{H}) \rightarrow \operatorname{Aut}\left(\mathcal{T}_{k}\right)
$$

such that for all $g \in \operatorname{Aut}(\mathcal{H}), \pi \pi^{*}(g)=g \pi \in \operatorname{Hom}\left(\mathcal{T}_{k}, \mathcal{H}\right)$.

Proof. This follows from the theory of covering maps (see, for example, [11]).

Let $\pi_{*}$ also denote the induced unitary isomorphism of vector spaces:

$$
\pi_{*}: V_{\mathcal{T}}^{n} \rightarrow V_{\mathcal{H}}^{n} .
$$

Proof of Theorem 9.1. Let $A_{n}=A_{n}(k)$ for each $n$. For each $n$, let $\iota: \operatorname{End}\left(V_{\mathcal{T}}^{n}\right) \rightarrow$ $\operatorname{End}\left(V_{\mathcal{H}}^{n}\right)$ be the injective $*$-morphism of algebras $\iota: F \mapsto \pi_{*} F \pi_{*}^{-1}$. It is obvious that $\iota\left(Q_{n}\right) \subset P_{n}$. Since $\pi$ preserves the trace weights, $\iota$ is trace-preserving. By Corollary 9.5 if $F \in Q_{n}$ commutes with $\operatorname{Aut}\left(\mathcal{T}_{k}\right)$, then $\iota(F)$ commutes with $\operatorname{Aut}(\mathcal{H})$ and hence with the $G$-action. Therefore, $\iota\left(A_{n}\right) \subset B_{n}$ for each $n$. It is obvious that $\iota$ is compatible with the inclusions $A_{n} \subset A_{n+1}$ and $B_{n} \subset B_{n+1}$. So we have an injective trace-preserving morphism of filtered algebras $\iota: \bigcup_{n} A_{n} \rightarrow \bigcup_{n} B_{n}$.

It remains to show that $\iota$ preserves the Jones projections. If $\rho$ is a rooted path of length $n$ on a graph, let $\rho(i)$ denote the $i$ th edge of $\rho, 1 \leq i \leq n$. To show that $\pi_{*}$ preserves Jones projections, it suffices to show that if $\rho$ is a rooted path of length $n$ on $\mathcal{T}$ and $\pi_{*}(\rho)=\mu$, then $\rho(n-1)=\rho(n) \Longleftrightarrow \mu(n-1)=\mu(n)$. Direction $\Longrightarrow$ is obvious. Assume $\mu(n-1)=\mu(n)$. Since $\mathcal{T}_{k}$ is a tree, $\rho(n-1)=\rho(n)$ iff the $(n-2)$ nd and $n$th vertices of $\rho$ are the same. Let

$$
\mu=v_{0} e_{1} v_{1} e_{2} \ldots e_{n-1} v_{n-1} e_{n-1} v_{n-2}
$$

and let

$$
\mu^{\prime}=v_{0} e_{1} v_{1} e_{2} \ldots e_{n-2} v_{n-2}
$$

be the truncation of $\mu$ by its last two edges. Clearly $\mu^{r}$ on $\mathcal{H}^{r}$ is homotopic to $\left(\mu^{\prime}\right)^{r}$. It follows that the lifts of $\mu$ and $\mu^{\prime}$ to $\mathcal{T}_{k}$ must end at the same vertex, since $\pi$ is a covering map (Proposition 9.2). Let $\rho^{\prime}$ be the truncation of $\rho$ by its last two edges. The lift of $\mu$ is $\rho$ and the lift of $\mu^{\prime}$ is $\rho^{\prime}$; hence $\rho^{\prime}$ and $\rho$ end at the same vertex. Therefore, the $(n-2)$ nd and $n$th vertices of $\rho$ are the same.

Proposition 9.6. The inclusion $\iota$ in Theorem 9.1 is not surjective.

Proof. Let $A_{n}=A_{n}(k)$ for each $n$. Let $\mathcal{T}_{k}^{n}$ be the subtree of $\mathcal{T}_{k}$ whose vertex set is those vertices whose distance from the root is at most $n$. Clearly $\mathcal{T}_{k}^{n}$ is stabilized by $\pi^{*}(G)$ and $\operatorname{Aut}\left(\mathcal{T}_{k}\right)$. Fix $n>p$. Let $G(n) \subset \operatorname{Aut}\left(\mathcal{T}_{k}^{n}\right)$ be the subgroup generated by the action of $\pi^{*}(G)$ on $\mathcal{T}_{k}^{n}$. We claim that it suffices to prove that $G(n)$ is a proper subgroup of $\operatorname{Aut}\left(\mathcal{T}_{k}^{n}\right)$. Assume this is so. Choose $N$ such that there exists a rooted path $\rho$ on $\mathcal{T}_{k}^{n}$ of length $N$ that ends at the root and covers $\mathcal{T}_{k}^{n}$. Let $F \in \operatorname{End}\left(V_{\mathcal{T}}^{N}\right)$ be the orthogonal projection to $\operatorname{Span}\left(\pi^{*}(G) \rho\right)$, the span of the $\pi^{*}(G)$ orbit of $\rho$. It is easy to check that $\pi_{*} F \pi_{*}^{-1} \in B_{N}$. By construction of $\rho$, the dimension of $\left(\operatorname{Span}\left(\pi^{*}(G) \rho\right)\right)$ is the order of $G(n)$. We have

$$
\operatorname{Aut}\left(\mathcal{T}_{k}\right)\left(\pi^{*}(G) \rho\right)=\operatorname{Aut}\left(\mathcal{T}_{k}\right) \rho=\operatorname{Aut}\left(\mathcal{T}_{k}^{n}\right) \rho .
$$

Again by construction of $\rho$, the dimension of $\operatorname{Span}\left(\operatorname{Aut}\left(\mathcal{T}_{k}^{n}\right) \rho\right)$ is the order of $\operatorname{Aut}\left(\mathcal{T}_{k}^{n}\right)$, which is greater than the order of $G(n)$ by assumption. Therefore, $\operatorname{Span}\left(\pi^{*}(G) \rho\right)$ is not stabilized by the action of $\operatorname{Aut}\left(\mathcal{T}_{k}\right)$, hence $F$ is not contained in $A_{N}$. Therefore, $\pi_{*} F \pi_{*}^{-1}$ is not in $\iota\left(A_{N}\right)$. Since $A_{N+m} \cap\left(\operatorname{End}\left(V_{\mathcal{T}}^{N}\right) \otimes 1^{m}\right)=A_{N} \otimes 1^{m}$ for each positive integer $m, \pi_{*} F \pi_{*}^{-1}$ is not in the image of $\iota$. 
So it suffices to show that $G(n)$ is a proper subgroup of $\operatorname{Aut}\left(\mathcal{T}_{k}^{n}\right)$. This is easy, because $G(n)$ can be generated by $p$ generators (by definition of $G$ ), but $\operatorname{Aut}\left(\mathcal{T}_{k}^{n}\right)$ requires $n$ generators. (Here is a simple proof: if $\sigma \in \operatorname{Aut}\left(\mathcal{T}_{k}^{n}\right)$ let $\sigma_{i}$ be the permutation given by the action of $\sigma$ on the set of vertices that are distance $i$ from the root. Let $\epsilon_{i}$ be the sign of $\sigma_{i}$. Then the map $\sigma \mapsto\left(\epsilon_{i}\right)_{1 \leq i \leq n}$ is a surjective group homomorphism onto $(\mathbb{Z} / 2 \mathbb{Z})^{n}$.)

\section{The Index 4 Case}

The subfactor $R_{U} \subset R$ has index 4 when $k=2$. It will be helpful to keep the following examples in mind. Let $[k]=\left\{x_{1}, x_{2}\right\}$. Denote elements of $S_{2}=\{(1),(12)\}$ with cycle notation, and let $S_{2}$ act on $[k]$ by its action on the subscripts of $x_{1}$ and $x_{2}$. Fix a positive integer $n$ and choose $p$ such that there is a permutation in $S_{p-1}$ of order $n$. Define actions of $[k]=\left\{x_{1}, x_{2}\right\}$ and $[p]=\left\{\alpha_{1}, \ldots, \alpha_{p}\right\}$ on each other as follows. Let $x_{1}$ act identically on $[p]$. Let $x_{2}$ fix $\alpha_{p}$ and act on $\left\{\alpha_{1}, \ldots, \alpha_{p-1}\right\}$ with order $n$. Let $\alpha_{1}, \ldots, \alpha_{p-1}$ act identically on $[k]$. We consider two cases.

Suppose $\alpha_{p}$ acts identically on $[k]$. It is easy to see that these actions arise from a permutation biunitary $U$. Namely, $U$ is given by

$$
U_{\alpha a}^{\beta b}=\delta_{\alpha, b(\beta)} \cdot \delta_{a, \beta(b)}
$$

for $\alpha, \beta \in[p]$ and $a, b \in[k]$. Denote this biunitary by $U(n,(1))$. In block form,

$$
U(n,(1))=\left[\begin{array}{cc}
I_{p} & 0 \\
0 & x_{2}
\end{array}\right]
$$

where $I_{p}$ denotes the $p \times p$ identity matrix and $x_{2}$ here denotes the $p \times p$ permutation matrix for the $x_{2}$-action on row vectors.

In the second case, $\alpha_{p}$ acts as (12) on $[k]$. Again there is a permutation biunitary, which we denote $U(n,(12))$, that gives rise to these actions. Let $x_{2}^{\prime}$ denote the $(p-1) \times(p-1)$ permutation matrix, acting on row vectors, of the restriction of the $x_{2}$ action to $\left\{\alpha_{1}, \ldots, \alpha_{p-1}\right\}$. Then

$$
U(n,(12))=\left[\begin{array}{cc}
\left(I_{p-1}, 0\right) & \left(0_{p-1}, 1\right) \\
\left(0_{p-1}, 1\right) & \left(x_{2}^{\prime}, 0\right)
\end{array}\right]
$$

where $U(n,(12))$ is written in block form and each block entry $(J, K)$ is a $p \times p$ block diagonal matrix with one $(p-1) \times(p-1)$ block $J$ and one $1 \times 1$ block $K$.

We will show that when $U=U(n,(1))$, we have $(\mathcal{H}, G)=\left(A_{2 n-1}^{(1)}, 1\right)$, and the principal graph is $A_{2 n-1}^{(1)}$; and when $U=U(n,(12))$, we have $(\mathcal{H}, G)=\left(A_{2 n-1}^{(1)}, S_{2}\right)$, and the principal graph is $A_{1}^{(2)}$, if $n=1$, and $D_{n+2}^{(1)}$, if $n \geq 1$. Moreover, we will show this is a complete set of principal graphs of Krishnan-Sunder subfactors of index 4 .

For the moment, fix any Krishnan-Sunder subfactor of index 4 arising from a permutation biunitary $U$. For $g \in S_{2}$, define

$$
[p]_{g}=\left\{\alpha \in[p]: \alpha \text { acts on }\left\{x_{1}, x_{2}\right\} \text { like } g\right\} .
$$

By Corollary 13 of [10], we may assume that

$$
\begin{array}{r}
x_{1} \text { acts identically on }[p], \\
{[p]_{(1)} \text { is nonempty. }}
\end{array}
$$

Lemma 10.1. (1) The action of $x_{2}$ on $[p]$ stabilizes $[p]_{(1)}$ and $[p]_{(12)}$.

(2) If $g \in S_{2}$ and $\alpha \in[p]_{g}$, then $\alpha$ acts on $\mathcal{F}$ as the diagonal action of $g$. 
Proof. To prove part 1, it suffices to prove $x_{2}$ stabilizes $[p]_{(1)}$. Suppose there exists $\alpha \in[p]_{(1)}$ such that $x_{2}(\alpha) \in[p]_{(12)}$. Let $x_{2}(\alpha)=\beta$. Since $\alpha\left(x_{2}\right)=x_{2}$ and $x_{2}(\alpha)=\beta, U_{\beta x_{2}}^{\alpha x_{2}}=1$. Also, $\beta\left(x_{1}\right)=x_{2}$ and $x_{1}(\beta)=\beta$; hence $U_{\beta x_{2}}^{\beta x_{1}}=1$. But then $U$ has two 1's in its $\left(\beta, x_{2}\right)$ column. Contradiction.

We now prove part 2] Let $w=a_{1} a_{2} \ldots a_{n} \in\left\{x_{1}, x_{2}\right\}^{n}$ and $g \in S_{2}$. By part 1 and (3.1),

$$
a_{1} a_{2} \ldots a_{r}\left([p]_{g}\right) \subset[p]_{g}
$$

for each $r, 1 \leq r \leq n$. Then by (3.3),

$$
\alpha\left(a_{1} a_{2} \ldots a_{n}\right)=g\left(a_{1}\right) g\left(a_{2}\right) \ldots g\left(a_{n}\right) .
$$

Proposition 10.2. Let $G$ and $\mathcal{H}$ be as defined in Sections 3 and 5 , Then:

(1) $\mathcal{H}$ is the Coxeter graph $A_{2 n-1}^{(1)}$, where $n$ is the order of the action of $x_{2}$ on $[p]$.

(2) $G$ is trivial if $[p]_{(12)}$ is empty, and $G \cong S_{2}$ otherwise.

Proof. 1. By Remark [5.9, $\mathcal{H}$ is a bipartite graph in which every vertex is adjacent to exactly two edges. Evidently, $\mathcal{H}$ must be $A_{\infty, \infty}$ or $A_{2 n-1}^{(1)}$ for some $n$. Let $n$ be the smallest integer such that $\left(x_{2} x_{1}\right)^{n} \sim e$, if such an $n$ exists. By construction of $\mathcal{H}$, if $n$ exists then $\mathcal{H} \cong A_{2 n-1}^{(1)}$. Otherwise, $\mathcal{H} \cong A_{\infty, \infty}$.

We must show that $n$ is the order of the $x_{2}$ action (which of course exists). Let $m$ be any positive integer. By (3.1), (3.3), and (10.3),

$$
\begin{array}{ll}
\alpha\left(\left(x_{2} x_{1}\right)^{m}\right)=\left(x_{2} x_{1}\right)^{m} & \text { if } \alpha \in[p]_{(1)} \\
\alpha\left(\left(x_{2} x_{1}\right)^{m}\right)=\left(x_{1} x_{2}\right)^{m} & \text { if } \alpha \in[p]_{(12)} \\
\left(x_{2} x_{1}\right)^{m}(\alpha)=x_{2}^{m}(\alpha) & \text { for all } \alpha \in[p] \\
\left(x_{1} x_{2}\right)^{m}(\alpha)=x_{2}^{-m}(\alpha) & \text { for all } \alpha \in[p]
\end{array}
$$

Note that the $G$-orbit of $\left(x_{2} x_{1}\right)^{m}$ is a nonempty subset of

$$
\left\{\left(x_{2} x_{1}\right)^{m},\left(x_{1} x_{2}\right)^{m}\right\} .
$$

So by (10.7), (10.8), and Remark 3.7. $\left(x_{2} x_{1}\right)^{m} \sim e$ iff $x_{2}^{m}$ acts identically on $[p]$. Therefore, $n$ is the order of the $x_{2}$-action.

2. Immediate from Lemma 10.1 and (10.4).

Proposition 10.3. The pairs $(\mathcal{H}, G)$ arising from permutation biunitaries $U$ with $k=2$ are exactly $\left(A_{2 n-1}^{(1)}, 1\right)$ and $\left(A_{2 n-1}^{(1)}, S_{2}\right), n=1,2,3, \ldots$ In particular, if $U=U(n,(1))$, then $(\mathcal{H}, G)=\left(A_{2 n-1}^{(1)}, 1\right)$, and if $U=U(n,(12))$, then $(\mathcal{H}, G)=$ $\left(A_{2 n-1}^{(1)}, S_{2}\right)$.

Proof. By Proposition 10.2, the pairs listed above are the only possible pairs $(\mathcal{H}, G)$. So it suffices to prove the second statement, which follows directly from Proposition 10.2 .

Corollary 10.4. The principal graphs arising from Krishnan-Sunder subfactors of index 4 are $A_{2 n-1}^{(1)}, n \geq 1$, and $D_{n}^{(1)}, n \geq 4$. In particular, if $U=U(n,(1))$, then the principal graph of $R_{U} \subset R$ is $A_{2 n-1}^{(1)}$; if $U=U(1,(12))$, then the principal graph of $R_{U} \subset R$ is $A_{1}^{(1)}$; and if $U=U(n,(12))$, with $n \geq 2$, then the principal graph of $R_{U} \subset R$ is $D_{n+2}^{(1)}$. 
Proof. We need only calculate $\Gamma$, using Theorem $[8.2$, from the pairs $(\mathcal{H}, G)$ listed in the previous proposition. The cases $(\mathcal{H}, G)=\left(A_{2 n-1}^{(1)}, 1\right)$ and $(\mathcal{H}, G)=\left(A_{1}^{(1)}, S_{2}\right)$ are both extreme cases, discussed by Krishnan and Sunder ([10], see the end of Section 5). The corresponding principal graphs are $A_{2 n-1}^{(1)}$ and $A_{1}^{(1)}$, respectively (see Remark 8.4). If $(\mathcal{H}, G)=\left(A_{2 n-1}^{(1)}, S_{2}\right), n \geq 2$, then Remark 8.3 applies. It is easy to verify that $\Gamma=D_{n+2}^{(1)}$. Let $\left\{*=v_{0}, v_{1}, \ldots, v_{2 n-1}\right\}$ be the vertex set of $A_{2 n-1}^{(1)},\left\{e_{1}, e_{2}, \ldots, e_{2 n}\right\}$ the edge set, and $\left\{v_{i-1}, v_{i}\right\}$ the endpoint set of the edge $e_{i}, 0 \leq i \leq 2 n$, taking indices of vertices $\bmod 2 n$. Then the action of $(12) \in G$ on $A_{2 n-1}^{(1)}$ is given by the vertex map $v_{i} \mapsto v_{2 n-i}$. Using the notation of Theorem 8.2 we may set $O=\left\{v_{0}, v_{1}, \ldots, v_{n}\right\}$. Given $v \in O$, if $v=v_{0}$ or $v=v_{n}$, then $G_{v}=S_{2}$. Otherwise $G_{v}$ is trivial. Thus, $v_{0}$ and $v_{n}$ each "split" into two vertices in $\Gamma$. Using the simple induction rules for $S_{1}$ and $S_{2}$, we obtain the graph $D_{n+2}^{(1)}$.

\section{An Infinite-Depth Krishnan-Sunder Subfactor}

Set $k=p=3$. Let $[k]=\{1,2,3\}$ and $[p]=\{\alpha, \beta, \gamma\}$. Consider the following biunitary matrix $U$ in $M_{p}(\mathbb{C}) \otimes M_{k}(\mathbb{C})$, constructed by Krishnan and Sunder (example $(15)$ in [10]):

$$
U=\left[\begin{array}{lllllllll}
1 & 0 & 0 & 0 & 0 & 0 & 0 & 0 & 0 \\
0 & 1 & 0 & 0 & 0 & 0 & 0 & 0 & 0 \\
0 & 0 & 0 & 0 & 0 & 1 & 0 & 0 & 0 \\
0 & 0 & 0 & 0 & 1 & 0 & 0 & 0 & 0 \\
0 & 0 & 0 & 1 & 0 & 0 & 0 & 0 & 0 \\
0 & 0 & 1 & 0 & 0 & 0 & 0 & 0 & 0 \\
0 & 0 & 0 & 0 & 0 & 0 & 0 & 0 & 1 \\
0 & 0 & 0 & 0 & 0 & 0 & 0 & 1 & 0 \\
0 & 0 & 0 & 0 & 0 & 0 & 1 & 0 & 0
\end{array}\right]
$$

We have written $U$ in block form, where blocks are indexed, in order, by $\alpha, \beta$, and $\gamma$, and each block is indexed, in order, by 1,2 , and 3 .

Krishnan and Sunder showed that $R_{U} \subset R$ is an irreducible subfactor of infinite depth, and characterized the principal graph in terms of finite dimensional representations of subgroups of $G$. We will obtain a simple proof of their result, based on our general theory, and show that the principal graph is not a tree.

The biunitary $U$ gives rise to the following actions of $[k]$ and $[p]$ on one another:

$$
\begin{aligned}
& \alpha: 1 \mapsto 1 \quad \beta: 1 \mapsto 2 \quad \gamma: 1 \mapsto 3 \\
& 2 \mapsto 2 \quad 2 \mapsto 1 \quad 2 \mapsto 2 \\
& 3 \mapsto 3 \quad 3 \mapsto 3 \quad 3 \mapsto 1
\end{aligned}
$$

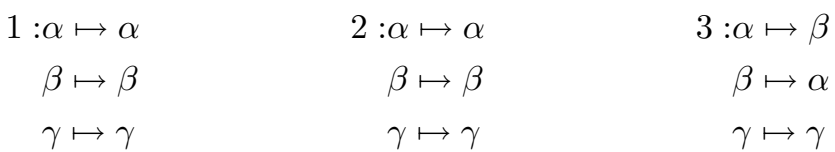

Lemma 11.1. For all integers $n \geq 0, \pi^{*}(G)$ acts transitively on the set of vertices in the nth generation of $\mathcal{T}_{3}$, where $\pi^{*}: \operatorname{Aut}(\mathcal{H}) \rightarrow \operatorname{Aut}\left(\mathcal{T}_{3}\right)$ is as in Corollary 9.5. 
Proof. The proof is by induction. Since only the root is in the 0th generation, the lemma is obvious for $n=0$. Let $I$ denote the set of vertices in the $(n-1)$ st generation of $\mathcal{T}_{3}$. Assume that $n>0$ and $\pi^{*}(G)$ acts transitively on $I$. We must prove that $\pi^{*}(G)$ acts transitively on the set of vertices in the $n$th generation of $\mathcal{T}_{3}$. For each vertex $v \in I, v$ has exactly 2 children, which we denote $v_{1}$ and $v_{2}$. We claim that it suffices to find $v \in I$ and $g \in \pi^{*}(G)$ such that $g\left(v_{1}\right)=v_{2}$. For suppose we have such a $v$ and $g$. Let $x, y \in I$. By the induction hypothesis there exist $h_{x}, h_{y} \in \pi^{*}(G)$ such that $h_{x}(x)=v$ and $h_{y}(y)=v$. Then either $h_{y}^{-1} h_{x}$ or $h_{y}^{-1} g h_{x}$ maps $x_{i}$ to $y_{j}$ for any $i, j \in\{1,2\}$. This proves the claim.

We now exhibit such a $v$ and $g$. Set

$$
\begin{gathered}
h=\gamma \alpha \beta \gamma \alpha \beta \gamma \alpha \beta, \\
g=\alpha h .
\end{gathered}
$$

Each element of $[p]$ is its own inverse as a permutation of $S_{k}$; hence $g \in G$. Let $v$ be the word of length $n-1$ of the form

$$
v=(121212 \ldots) 3 .
$$

Since $v$ is a reduced word of length $n-1, v$ is a vertex in the $(n-1)$ st generation of $\mathcal{T}_{3}$. The two children of $v$ are

$$
v_{1}=(121212 \ldots) 31
$$

and

$$
v_{2}=(121212 \ldots) 32 .
$$

We must show that $g\left(v_{1}\right)=v_{2}$.

We claim that $h$ acts identically on $\mathcal{F}$. It is easy to check that $\alpha \beta$ acts on $\mathcal{F}$ as the diagonal action of the cycle $(12) \in S_{3}$ and $\gamma$ acts on $\mathcal{F}$ as the diagonal action of the cycle $(13) \in S_{3}$. The claim follows since $(13)(12)(13)(12)(13)(12)$ equals the identity in $S_{3}$.

It is also easy to check that $\alpha\left(v_{1}\right)=v_{2}$. Therefore, $g\left(v_{1}\right)=\alpha h\left(v_{1}\right)=\alpha\left(v_{1}\right)=v_{2}$. So we are done.

Proposition 11.2. The graph morphism $\pi: \mathcal{T}_{3} \rightarrow \mathcal{H}$, as defined in Section 9 , is an isomorphism.

Proof. Since $\pi$ is a covering map (Proposition 9.2), it suffices to show that the inverse image of the root vertex of $\mathcal{H}$ contains only the root vertex of $\mathcal{T}_{3}$. In other words, we must show that if $w$ is a reduced word in $\mathcal{F}$ of positive length $n$, then $\pi(w)$ is not the root of $\mathcal{H}$. By Lemma 11.1 there exists $g \in G$ such that $g(w)$ is the word of length $n$ of the form $w^{\prime}=3(121212 \ldots)$. It is easy to check that $w^{\prime}(\alpha)=\beta$. So $w^{\prime}$ does not act identically on $[p]$, hence $w^{\prime}$ does not act identically on $[p]^{2}$, hence $w^{\prime} \nsim e$, and hence $w \nsim e$. Therefore, $\pi(w)$ is not the root of $\mathcal{H}$.

Remark 11.3. We can apply Theorem 8.2 to $(\mathcal{H}, G)$ to obtain the description of the principal graph given by Krishnan and Sunder in Theorem 37 of 10. Let $*=v_{0}, v_{1}, v_{2}, v_{3}, \ldots$ be a sequence of vertices in $\mathcal{H} \cong \mathcal{T}_{3}$, such that $v_{n}$ neighbors $v_{n+1}$ and the distance from $v_{n}$ to the root is $n$. By Lemma 11.1, $O=\left\{v_{n}\right\}_{0}^{\infty}$ is a set of representatives of the $G$-orbits in the vertex set of $\mathcal{H}$. Let $G_{n}=G_{v_{n}}$, the subgroup of $G$ that fixes $v_{n}$, for each $n$. It is easy to verify that $(\mathcal{H}, G)$ satisfies the hypotheses of Remark 8.3 Let us apply Theorem 8.2. The vertex set of $\Gamma^{0}$ is $\amalg_{n} \hat{G}_{n}$. Clearly $G_{n+1} \subset G_{n}$ for each $n$. So if $\lambda_{n} \in \hat{G}_{n}$ and $\lambda_{n+1} \in \hat{G}_{n+1}$, then the 
number of edges between $\lambda_{n}$ and $\lambda_{n+1}$ is $\operatorname{dim}\left(\operatorname{Hom}\left(\lambda_{n+1}, \operatorname{Res}_{G_{n+1}}^{G_{n}} \lambda_{n}\right)\right)$. Finally, $\Gamma$ is the connected component of $\Gamma^{0}$ containing $1_{*}$.

We now show that $\Gamma$ is not a tree, contrary to Krishnan and Sunder's expectations in [10. Let $\mathcal{T}_{3}(2)$ be the subtree of $\mathcal{T}_{3}$ within radius 2 of the root. It is the tree with 10 vertices in which the root vertex has 3 children and each of those children has 2 children. For $n=0,1$, and 2, let $H_{n} \subset \operatorname{Aut}\left(\mathcal{T}_{3}(2)\right)$ be the subgroup generated by the action of $G_{n}$ on $\mathcal{T}_{3}$, restricted to $\mathcal{T}_{3}(2)$. Each $H_{n}$ is a quotient of $G_{n}$, and there is a natural injection $\hat{H}_{n} \rightarrow \hat{G}_{n}$ that commutes with Res. Thus, if we replace $\mathcal{H}$ by $\mathcal{T}_{3}(2)$ and $G_{n}$ by $H_{n}$ in Definition 8.1 we obtain a subgraph of $\Gamma^{0}$, which we denote $\Gamma^{0}(2)$. Let $\Gamma(2)$ be the corresponding subgraph of $\Gamma$.

The vertices in the second generation of $\mathcal{T}_{3}(2)$ are $12,13,21,23,31$, and 32. Number these 1 through 6 respectively, and consider the image of $H_{n}$ in $S_{6}$, for $n=0,1$, and 2. It is easy to verify that $\alpha \mapsto(56), \beta \mapsto(13)(24)$, and $\gamma \mapsto$ (16)(25)(34). A calculation using GAP ([1]) shows that $\left|H_{0}\right| \geq 48$. But, as will be obvious below, $\operatorname{Aut}\left(\mathcal{T}_{3}(2)\right)$ has order 48 , so $H_{0}=\operatorname{Aut}\left(\mathcal{T}_{3}(2)\right)$.

It is well known that the automorphism groups of trees such as $\mathcal{T}_{3}(2)$ are wreath products of permutation groups. We have

$$
\begin{array}{clccc}
H_{0}=\operatorname{Aut}\left(\mathcal{T}_{3}(2)\right) & \cong & S_{3} \prec S_{2} & = & S_{3} \ltimes\left(S_{2} \times S_{2} \times S_{2}\right) \\
H_{1} & \cong & \left(S_{2} \prec S_{2}\right) \times S_{2} & = & \left(S_{2} \ltimes\left(S_{2} \times S_{2}\right)\right) \times S_{2} \\
H_{2} & \cong & \left(S_{2} \prec S_{2}\right) & = & S_{2} \ltimes\left(S_{2} \times S_{2}\right)
\end{array}
$$

with the inclusion maps

$$
\begin{aligned}
H_{1} & \rightarrow H_{0} \\
\left(\sigma\left(g_{1}, g_{2}\right), g_{3}\right) & \mapsto \sigma\left(g_{1}, g_{2}, g_{3}\right)
\end{aligned}
$$

where $g_{i}, \sigma \in S_{2}$ and $\sigma \in S_{3}$, via the usual inclusion $S_{2} \subset S_{3}$;

$$
\begin{aligned}
H_{2} & \rightarrow H_{1} \\
\sigma\left(g_{1}, g_{2}\right) & \mapsto\left(\sigma\left(g_{1}, g_{2}\right),(1)\right)
\end{aligned}
$$

where $\sigma, g_{i} \in S_{2}$ and (1) is the identity element of $S_{2}$.

The representation theory of these groups is well known ([5]). The irreducible representations of $S_{n} \prec S_{m}$ are in bijection with the set of objects given by: a choice of $r$ distinct irreducible representations $\nu_{i} \in \hat{S}_{m}, 1 \leq i \leq r$; positive integer weights $n_{i}, 1 \leq i \leq r$, such that $\sum^{r} n_{i}=n$; and a choice of $\mu \in\left(\prod^{r} S_{n_{i}}\right)^{r}$. The object is defined up to permutations of the $\nu_{i}$ (with the corresponding permutation of weights, etc.). We denote the corresponding representation by $(\mu)\left(\otimes^{r} \nu_{i}^{n_{i}}\right)$. The restriction rules are:

$$
\operatorname{Res}_{H_{2}}^{H_{1}}\left((\mu)\left(\bigotimes \nu_{i}^{n_{i}}\right) \otimes \nu\right)=\operatorname{dim}(\nu) \cdot(\mu)\left(\bigotimes \nu_{i}^{n_{i}}\right)
$$

where $\mu, \nu_{i} \in \hat{S_{2}}$ and $\sum n_{i}=2$;

$$
\operatorname{Res}_{H_{1}}^{H_{0}}(\mu)\left(\otimes^{r} \nu_{i}^{n_{i}}\right)=\bigoplus_{j=1}^{r}\left(\operatorname{Res}_{\prod S_{n_{i}^{\prime}}}^{\prod S_{n_{i}}} \mu\right)\left(\bigotimes^{r} \nu_{i}^{n_{i}^{\prime}}\right) \otimes \nu_{j}
$$

where $\mu \in \hat{S_{3}}, \nu_{i} \in \hat{S_{2}}, \sum^{r} n_{i}=3$, and

$$
n_{i}^{\prime}=n_{i}-\delta_{i, j} .
$$

Theorem 11.4. The principal graph of $R_{U} \subset R$ is not a tree. 
Proof. By the previous discussion it suffices to show that $\Gamma(2)$ contains a cycle. Let $1_{1}$ denote the trivial representation of the trivial group; let $1_{2}$ and $-1_{2}$ denote the trivial and alternating representations of $S_{2}$; and let $1_{3}, \nu_{3}$, and $-1_{3}$ denote the trivial, standard, and alternating representations of $S_{3}$. Let $\lambda_{i}, 0 \leq i \leq 9$, be the following elements, respectively:

$$
\begin{aligned}
\left(1_{2} \otimes 1_{1}\right)\left(1_{2}^{2} \otimes-1_{2}\right) & \in \hat{H}_{0} \\
\left(1_{1} \otimes 1_{1}\right)\left(1_{2} \otimes-1_{2}\right) \otimes 1_{2} & \in \hat{H}_{1} \\
\left(-1_{2} \otimes 1_{1}\right)\left(1_{2}^{2} \otimes-1_{2}\right) & \in \hat{H}_{0} \\
\left(-1_{2}\right)\left(1_{2}^{2}\right) \otimes-1_{2} & \in \hat{H}_{1} \\
\left(-1_{2}\right)\left(1_{2}^{2}\right) & \in \hat{H}_{2} \\
\left(-1_{2}\right)\left(1_{2}^{2}\right) \otimes 1_{2} & \in \hat{H}_{1} \\
\left(\nu_{2}\right)\left(1_{2}^{3}\right) & \in \hat{H}_{0} \\
\left(1_{2}\right)\left(1_{2}^{2}\right) \otimes 1_{2}=1_{H_{1}} & \in \hat{H}_{1} \\
\left(1_{2}\right)\left(1_{2}^{2}\right)=1_{H_{2}} & \in \hat{H}_{2} \\
\left(1_{2}\right)\left(1_{2}^{2}\right) \otimes-1_{2} & \in \hat{H}_{1}
\end{aligned}
$$

Then the $\lambda_{i}, 0 \leq i \leq 9$, are vertices of $\Gamma^{0}(2)$, and it is straightforward to check that there is an edge between $\lambda_{i}$ and $\lambda_{i+1}$ for each $i$, taking indices modulo 10 . Therefore, $\Gamma^{0}(2)$ contains a cycle. Finally, $\lambda_{7}$, the trivial representation of $H_{1}$, is clearly connected to the trivial representation of $H_{0}$, so $\Gamma(2)$ contains a cycle.

\section{REFERENCES}

1. The GAP Group, Aachen, St. Andrews, GAP - Groups, Algorithms, and Programming, Version 4, 1998, (http://www-gap.dcs.st-and.ac.uk/ gap).

2. B. Bhattacharyya, Krishnan-Sunder subfactors and a new countable family of subfactors related to trees, Ph.D. thesis, UC Berkeley, 1998.

3. Chris Godsil and Gordon Royle, Algebraic graph theory, Springer-Verlag, New York, 2001.

4. F. Goodman, P. de la Harpe, and V. F. R. Jones, Coxeter graphs and towers of algebras, MSRI Publications, vol. 14, Springer, 1989. MR 91c:46082

5. Gordon James and Adalbert Kerber, The representation theory of the symmetric group, Encyclopedia of Mathematics and its Applications, vol. 16, Addison-Wesley Publishing Co., Reading, Mass., 1981, with a foreword by P. M. Cohn, with an introduction by Gilbert de B. Robinson. MR 83k:20003

6. Jonathan L. Gross and Thomas W. Tucker, Topological graph theory, John Wiley \& Sons Inc., New York, 1987. MR 88h:05034

7. U. Haagerup and J. Schou, Some new subfactors of the hyperfinite II $I_{1}$ subfactor, 1989, preprint.

8. V. F. R. Jones, Index for subfactors, Invent. Math. 72 (1983), 1-25. MR 84d:46097

9. V. F. R. Jones and V. S. Sunder, Introduction to subfactors, London Mathematical Society Lecture Note Series, vol. 234, Cambridge University Press, 1997. MR 98h:46067

10. U. Krishnan and V. S. Sunder, On biunitary permutation matrices and some subfactors of index 9, Trans. Amer. Math. Soc. 348 (1996), no. 12, 4691-4736. MR 97c:46077

11. W. S. Massey, Algebraic topology: An introduction, ch. 6, Springer-Verlag, 1977. MR 56:6638

12. A. Ocneanu (Lecture Notes by Y. Kawahigashi), Quantum symmetry, differential geometry of finite graphs and classification of subfactors, 1990, University of Tokyo Seminar Notes.

13. Adrian Ocneanu, Quantized groups, string algebras and Galois theory for algebras, Operator algebras and applications, Vol. 2, London Math. Soc. Lecture Note Ser., vol. 136, Cambridge Univ. Press, Cambridge, 1988, pp. 119-172. MR 91k:46068 
14. S. Popa, Orthogonal pairs of *-subalgebras in finite von Neumann algebras, J. Operator Theory 9 (1983), no. 2, 253-268. MR 84h:46077

15. Classification of subfactors: the reduction to commuting squares, Invent. Math. 101 (1990), no. 1, 19-43. MR 91h:46109

16. Classification of amenable subfactors of type II, Acta Math. 172 (1994), no. 2, 163255. MR 95f:46105

17. - An axiomatization of the lattice of higher relative commutants of a subfactor, Invent. Math. 120 (1995), 427-445. MR 96g:46051

18. V. S. Sunder, A model for AF algebras and a representation of the Jones projections, J. Operator Theory 18 (1987), 289-301. MR 89e:46079

19. Hans Wenzl, Hecke algebras of type $A_{n}$ and subfactors, Invent. Math. 92 (1988), no. 2, 349383. MR 90b:46118

Elance, 820A Kifer Rd., Sunnyvale, California 94086

E-mail address: Bina_Bhattacharyya_91@post.harvard.edu 